\title{
Natural Resource Management for Nonlinear Stochastic Biotic-Abiotic Ecosystems: Robust Reference Tracking Control Strategy Using Limited Set of Controllers
}

\author{
Y. P. Lin and B. S. Chen* \\ Laboratory of Control and Systems Biology, National Tsing Hua University, Hsinchu 30013, Taiwan
}

Received 17 December 2013; revised 31 July 2014; accepted 18 August 2014; published online 15 July 2015

\begin{abstract}
The management of natural resources has become an important subject because of the increasing population. To meet human requirements for resources such as food and water, many control strategies have been proposed to improve biomass production and nutrient supply. However, implementing these strategies may be limited by the expense of large numbers of control devices and by the effects of stochastic perturbations. In this study, a robust reference tracking control strategy for the natural resource management of nonlinear stochastic biotic-abiotic ecosystems is proposed, using a limited set of controllers, to regulate the systematic dynamics achieving a desired reference trajectory under the influence of intrinsic fluctuations and environmental disturbances. To simplify the design procedure and make the robust reference tracking control strategy more feasible, we propose a fuzzy stochastic partial differrential equations system to represent the ecosystem, itself approximated by a fuzzy stochastic spatial state space model, based on a finite difference scheme. This allows replacement of a complex Hamilton-Jacobi integral inequality by an equivalent set of local linear matrix inequalities which can be easily solved. We verify the efficiency of the proposed approach using a nonlinear stochastic biomass-nutrient control example and compare the robust tracking control performance for different arrangements of control devices. Managers may select appropriate tracking control schemes based on this comparison. The robust reference tracking control strategy can be applied not only to agricultural systems but also to biophysical systems in ecological conservation, ecosystem restoration or engineering.
\end{abstract}

Keywords: natural resource management, spatial state space model, robust reference tracking control, T-S fuzzy interpolation, finite

difference scheme

\section{Introduction}

Ecosystems consist of biotic populations and an abiotic environment interacting with each other. Both natural and artificial ecosystems provide people with fundamental goods and services. These ecological services, such as nutrient cycling, food and water provision or climate regulation, are equally important for sustaining the ecosystems themselves. Because of the increasing human population and associated effects (e.g., pollution and climate change), natural resource management (NRM) has however become a central issue for sustainably supporting both present and future generations, especially with respect to food and fresh water requirements. Total human population is projected to exceed 9 billion in 2050 (US$\mathrm{CB}, 2011$ ) (Figure 1), raising the question of how enough food can be produced to feed this population. Sixty percent of the world's food production comes from rain-fed agriculture (WBCSD and IUCN, 2009), but the full potential of rain-fed agricul-

\footnotetext{
${ }^{*}$ Corresponding author. Tel.: +886-3-573-1155; fax: +886-3-571-5971

E-mail address: bschen@ee.nthu.edu.tw (B. S. Chen).
}

ISSN: $1726-2135$ print/1684-8799 online

(C) 2016 ISEIS All rights reserved. doi:10.3808/jei.201400286 ture is never achieved because water availability is affected by climatic events such as droughts. Even in irrigated agriculture, irrigation efficiency is only $38 \%$ globally, since $\sim 62 \%$ of the water delivered to fields is lost as runoff or to drainage (FAO, 2003) (Table 1). Since large amounts of water are required for food production, improvement of the productivity of existing farmland and the efficiency of water usage is an urgent priority.

Many strategies have recently been proposed to improve food production and water use efficiency for human needs (Evans et al., 1991; Burke et al., 1999; Ahuja et al., 2008; Boutraa, 2010). Artificial nutrient supply and pest control are the principal methods of increasing biomass yield. In intensive agricultural production, nutrient supply is increased by the application of fertiliser and irrigation water. This is a basic control input to improve plant growth. Pest control is carried out through the application of chemical pesticide, biological control agents or herbivore exclosures. This is an alternative control input to decrease plant mortality caused by disease or grazing. However, growing global awareness of environmental problems and concern for human health have led to increasing demand for organic foods and pesticide-free products, and decreasing use of chemical fertilisers and pesticides (Matthews, 1999; Tilman et al., 2002; Pearce and Koundouri, 2003). Human activities and global climate change have also reduced 


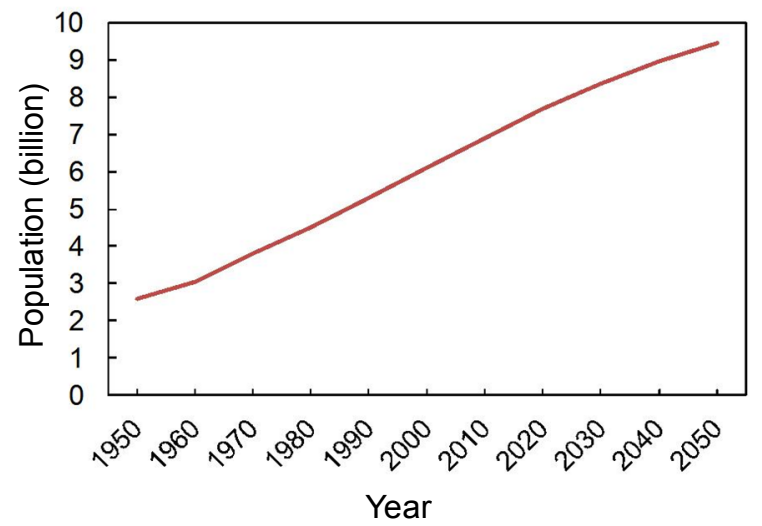

*Note: Source: U.S. Census Bureau, International Data Base, 2011

Figure 1. World population: $1950 \sim 2050$. It will be projected to exceed 9 billion people in $2050^{*}$.

the amount of water available for irrigation in some regions (Rosegrant et al., 2002; Fereres and Soriano, 2007; Hanjra and Qureshi, 2010; Monsef and Abahussain, 2013). More than 40\% of the global land area is classified as arid or semi-arid (Boutraa, 2010). In these water-poor areas, water is the most important limiting factor in agricultural production, thus improving irrigation efficiency is important in these areas. The use of irrigation water, chemical fertiliser and pesticide to improve crop yield requires innovative and sustainable research. Techniques for improving water use efficiency and crop yield have been developed using engineering approaches (Wallace, 2000) or innovative subsurface irrigation instead of traditional flood irrigation (Batchelor et al., 1996). Systematic perspectives that apply systems theory to NRM have also been used to develop theoretical strategies for improving crop yield and water use efficiency (Amir et al., 1991; Bouman et al., 2005).

Growth in the demand for natural resources such as food and water will be concentrated in arid and semi-arid environments in many developing countries. In these areas, population growth is generally high, which increases the need to improve crop yield and water use efficiency. However, stochastic perturbations such as alien species invasions or variability in irrigation water availability caused by changes in evaporation, transpiration or rainfall make farming in arid areas an unpredictable and high-risk activity. Ensuring that crop yield is maintained at a desired level despite stochastic perturbations is therefore a necessity for many developing countries. Strategies involving a robust reference tracking control, which would help to manipulate crop yield and regulate irrigation water in arid and semi-arid areas, are essential for NRM aimed at desired agricultural production levels. This strategy could also be used in ecological conservation and restoration to protect and restore land, water, animals, plants and air quality, or in ecological engineering to design, monitor, and construct artificial ecosystems (Bergen et al., 2001). To simplify the theoretical analysis of ecosystems, early studies have mainly focused on the interactions of uniform (well-mixed) biotic populations and abiotic environments, which can be represented by deterministic ordinary differential equations (ODEs) (Gurney and Nisbet, 1998; Cale et al., 2009; Thébault and Fontaine, 2010). However in the real world the spatial distribution of natural resources is mostly heterogeneous, and their spatiotemporal systematic dynamics are generally nonlinear associated with stochastic intrinsic fluctuations and local environmental disturbances. Systems of nonlinear stochastic partial differential equations (PDEs) are therefore more suitable for representing real biotic-abiotic interactions over space and time. In other words, the design of robust reference tracking controls for nonlinear stochastic biotic-abiotic ecosystems should take into account not only the effects of stochastic intrinsic fluctuations and local environmental disturbances, but also systematic dynamics that depend on space and time.

The design of robust reference tracking controls for nonlinear stochastic PDEs systems requires solving a complex Hamilton-Jacobi integral inequality (HJII). This is more difficult than solving a Hamilton-Jacobi inequality (HJI) to design a robust reference control for nonlinear stochastic ODEs systems. At present, there are no direct analytic or numerical solutions for HJII or HJI except for some simple cases. The T-S fuzzy method, which interpolates several local linear models to approximate a nonlinear system, has seen recent and widespread use in solving HJI problems for nonlinear systems described by ODEs (Tanaka and Sugeno, 1992; Chen et al., 1996; Tanaka et al., 1996, 1998; Chen et al., 1999, 2000; Tseng et al., 2001; Nguang and Shi, 2003; Tanaka and Wang, 2004). Based on the method of Galerkin, which is used to derive a set of nonlinear ODEs approximating a PDEs system (Wu and $\mathrm{Li}$ 2008; Yuan et al., 2008), several control design schemes have been proposed to stabilise nonlinear PDEs systems. A fuzzy infinite-dimensional state space model based on the Galerkin method has been proposed to represent a nonlinear PDEs system with some truncation error (Chen and Chang, 2009). An alternative method, the finite difference scheme (Strikwerda, 2004), has also been widely applied to obtain the numerical solutions of PDEs, and a fuzzy tracking control for nonlinear PDEs systems with environmental disturbances has been proposed based on this scheme (Chang and Chen, 2010). However, many control methods remain theoretical, as it is very difficult and probably unrealistic to design a controller for the entire PDEs system. The feasibility of implementing any control strategy over a large area is generally limited by the expense of applying a large number of control devices. Applying a limited number of control devices at some locations instead of a strategy to control the entire system is more realistic. We therefore use a limited set of controllers in designing a robust reference tracking control by combining a fuzzy interpolation approach with a finite difference scheme for nonlinear stochastic PDEs systems with random intrinsic and external perturbations. This control method can be applied to nonlinear stochastic bioticabiotic ecosystems and other biophysical systems over space and time. For practical applications, we also validate the performance of different distribution control strategies using a limited set of controllers. This may help managers to effectively reduce the usage amount of control devices, and provide an optimal sorting method for control devices.

We use a fuzzy interpolation approach and a finite difference scheme, taking into account practical design require- 
Table 1. Water Use Efficiency in 1998 and 2030 (Predicted) in 93 Developing Countries (FAO 2003)*

\begin{tabular}{lllllll}
\hline & $\begin{array}{l}\text { Sub-Saharan } \\
\text { Africa }\end{array}$ & $\begin{array}{l}\text { Latin } \\
\text { America }\end{array}$ & $\begin{array}{l}\text { Near East \& } \\
\text { North Africa }\end{array}$ & $\begin{array}{l}\text { South } \\
\text { Asia }\end{array}$ & $\begin{array}{l}\text { East } \\
\text { Asia }\end{array}$ & $\begin{array}{l}\text { All } \\
\text { countries }\end{array}$ \\
\hline \multicolumn{7}{c}{ Water use efficiency in irrigation (\%) } \\
\hline 1998 & 33 & 25 & 40 & 44 & 33 & 38 \\
2030 & 37 & 25 & 53 & 49 & 35 & 42 \\
\hline
\end{tabular}

"Source: FAO, 2003.

ments (minimising the complexity of nonlinear interactions, attenuating the effect of environmental disturbances, and limiting the number of control devices), to select a suitable space partition size and numbers of fuzzy local linear systems. We use these to represent a nonlinear stochastic biotic-abiotic ecosystem with a fuzzy spatial state space model, with some approximation and truncation error. We assume that the effects of system spatial dynamics (i.e. diffusion and advection) have uniform influences over the unit grid-space. Based on this fuzzy spatial state space model, we propose a robust reference tracking control strategy to control the ecosystem and achieve the desired reference trajectory tracking in spite of the effect of stochastic intrinsic and external perturbations and errors resulting from approximation and truncation. In this way, a complex HJII (or HJI) can be replaced by an equivalent linear matrix inequalities (LMIs) set, which can be solved easily using the LMI toolbox in Matlab.

In this study, we design a robust reference tracking control for nonlinear stochastic biotic-abiotic ecosystems for NRM using a limited set of controllers. In section 2, we model a nonlinear stochastic PDEs system to describe such an ecosystem, including random intrinsic fluctuations and environmental disturbances, and propose a robust reference tracking control to attenuate the effect of random disturbances on the tracking error. In section 3, we represent the ecosystem as a fuzzy spatial state space model to overcome nonlinearity and partial differentiation in the design of the robust reference tracking control, using the following procedures: (a) T-S fuzzy interpolation is used to propose a fuzzy stochastic PDEs system to represent the ecosystem by interpolating several local linear stochastic PDEs systems. (b) A finite difference scheme is used to discretize the continuous spatial domain by dividing it into several uniform grids, and a finite difference operator is proposed to represent the partial differential operator. This allows representation of a fuzzy stochastic PDEs system by an equivalent fuzzy spatial state space model. (c) Based on the fuzzy spatial state space model, a fuzzy tracking control design is proposed to achieve the best possible robust tracking, i.e. attenuating the effects of intrinsic fluctuation, environmental disturbance, fuzzy approximation error and truncation error on tracking error. (d) Finally, the control gain for the robust fuzzy tracking control design is obtained by solving an equivalent LMIs-constrained optimisation problem for the ecosystem, using the LMI toolbox in Matlab. In section 4, we validate the robust fuzzy tracking control using a numerical example, and compare and validate the performance of different distribution control strategies. Section 5 is devoted to the discussion of the results.

\section{Robust Reference Tracking Control Strategy of Nonlinear Stochastic Biotic-Abiotic Ecosystems}

The biophysical interactions of nonlinear stochastic bioticabiotic ecosystems can be described using abiotic and biotic variables whose dynamics are dependent on their two-dimensional spatial position $x=\left[x_{1}, x_{2}\right] \in \Omega$ and time $t \in\left[0, t_{f}\right]$. In general, ecological patterns such as the heterogeneous distribution of species or substances can be induced by Turing instability, differential flow instability or random environmental fluctuations (Satnoianu et al., 2000; Tilman et al., 2002; Huang and Diekmann, 2003). The heterogeneous spatial distribution of these biotic and abiotic natural resources can be represented by the following reaction-diffusion-advection equations (Bouman et al., 2005), which incorporate intrinsic parameter fluctuations and environmental disturbances:

$$
\begin{aligned}
& \frac{\partial \chi(x, t)}{\partial t}=\left(f(\chi(x, t))+f_{\omega}(\chi(x, t)) n(x, t)\right)+D \nabla^{2} \chi(x, t)+ \\
& V \nabla \chi(x, t)+d(x, t)
\end{aligned}
$$

where the state vector $\chi(x, t)=\left[\chi_{l}(x, t), \ldots, \chi_{n}(x, t)\right]^{T} \in \mathfrak{R}^{n}$ contains all $n$ biotic and abiotic state variables. In (1), the reaction term $\left(f(\chi(x, t))+f_{\omega}(\chi(x, t)) n(x, t)\right)$ denotes the nonlinear bioticabiotic interaction $f(\chi(x, t)) \in \mathfrak{R}^{n}$ with the random intrinsic parameter fluctuation $f_{\omega}(\chi(x, t)) n(x, t) \in \mathfrak{R}^{n}$, where $n(x, t) \in \mathfrak{R}^{1}$ is stationary white noise with zero mean and unit variance. The diffusion term $D \nabla^{2} \chi(x, t) \in \mathfrak{R}^{n}$ denotes the spreading of state variables with diffusivity $D=\operatorname{diag}\left(d_{1}, \ldots, d_{n}\right) \in \mathfrak{R}^{n \times n}$ and the advection term $V \nabla \chi(x, t) \in \mathfrak{R}$ denotes the movement of state variables with velocity $V=\operatorname{diag}\left(v_{1}, \ldots, d_{n}\right) \in \mathfrak{R}^{n \times n}$. The final term $d(x, t) \in \mathfrak{R}^{n}$ denotes the bounded spatiotemporal environmental disturbances. In the two-dimensional space case, the differential operators $\nabla$ and $\nabla^{2}$ are defined as follows (Gurney and Nisbet, 1998):

$\nabla \chi(x, t) \equiv \sum_{k=1}^{2} \frac{\partial}{\partial x_{k}} \chi(x, t), \nabla^{2} \chi(x, t) \equiv \sum_{k=1}^{2} \frac{\partial^{2}}{\partial x_{k}^{2}} \chi(x, t)$

where $\partial \chi(x, t) / \partial x_{k} \equiv\left[\partial \chi_{I}(x, t) / \partial x_{k}, \ldots, \partial \chi_{n}(x, t) / \partial x_{k}\right]^{T} \in \mathfrak{R}^{n}$, $\partial^{2} \chi(x, t) / \partial x_{k}{ }^{2} \equiv\left[\partial^{2} \chi_{1}(x, t) / \partial x_{k}{ }^{2}, \ldots, \partial^{2} \chi_{n}(x, t) / \partial x_{k}{ }^{2}\right]^{T} \in \mathfrak{R}^{n}, k=$ 1,2 . The initial value is given by $\chi(x, 0)$ and the boundary condition is given by the Dirichlet boundary condition, $\chi(x, t)$ $=0$ on $\partial \Omega$, or the Neumann boundary condition, $\partial \chi(x, t) / \partial x=$ 0 on $\partial \Omega$.

For the purpose of system analysis and control design, the reaction-advection-diffusion equations in the above ecosystem 
(1) can be represented by the following Ito stochastic system (Øksendal, 2003):

$$
\begin{aligned}
& d \chi(x, t)=\left(f(\chi(x, t))+D \nabla^{2} \chi(x, t)+V \nabla \chi(x, t)+d(x, t)\right) d t+ \\
& f_{\omega}(\chi(x, t)) d \omega(x, t)
\end{aligned}
$$

where $\omega(x, t)$ with $d \omega(x, t)=n(x, t)$ denotes a standard Wiener process, or Brownian motion. By limiting the set of controllers used for the tracking control design (i.e. control devices are only set up at $p$ locations), the ecosystem in (3) can be rewritten as follows:

$$
\begin{aligned}
& d \chi(x, t)=\left(f(\chi(x, t))+D \nabla^{2} \chi(x, t)+V \nabla \chi(x, t)+g(x) u(x, t)+\right. \\
& d(x, t)) d t+f_{\omega}(\chi(x, t)) d \omega(x, t)
\end{aligned}
$$

where the control input set up at $p$ point locations is defined as $u(x, t)=\left[u\left(x^{l}, t\right)^{T}, \ldots, u\left(x^{p}, t\right)^{T}\right]^{T} \in \mathfrak{R}^{n p}$. The control influence function $g(x)$ incorporates $p$ point locations, i.e. $g(x)=$ $\left[g\left(x^{l}\right), \ldots, g\left(x^{p}\right)\right] \in \mathfrak{R}^{n \times n p}$ in which $g\left(x^{i}\right)=I_{n}$, to influence the $n$ state variables at location $x^{i} \in x, i=1, \ldots, p$.

To manage natural resources for human needs, a management strategy must control the state variables to track a desired trajectory, such as an annual production plan. The reference model incorporating a desired dynamic response is described as below, following the Ito formula (Øksendal, 2003):

$$
d \chi_{r}(x, t)=\left(A_{r} \chi_{r}(x, t)+D_{r} \nabla^{2} \chi_{r}(x, t)+V_{r} \nabla_{r}(x, t)+r(x, t)\right) d t
$$

where the reference state vector $\chi_{r}(x, t)=\left[\chi_{r 1}(x, t), \ldots, \chi_{r n}(x\right.$, $t)]^{T} \in \mathfrak{R}^{n}$.. The linear reference matrix $A_{r} \in \mathfrak{R}^{n \times n}$ is specified with negative eigenvalues to guarantee the stability of the reference model to achieve a desired transient reference trajectory. The bounded reference input signal $r(x, t) \in \mathfrak{R}^{n}$ is specified to achieve a desired steady state reference trajectory according to the practical requirtment on state variables. The diagonal matrices $V_{r} \in \mathfrak{R}^{n \times n}$ and $D_{r} \in \mathfrak{R}^{n \times n}$ can also be specified if required. To allow the ecosystem in (4) to track the reference model in (5), a robust reference tracking control strategy is given as the following nonlinear error state feedback design:

$$
u(x, t)=K(\tilde{\chi}(x, t))
$$

where $\tilde{\chi}(x, t)=\chi(x, t)-\chi_{r}(x, t)$ denotes the tracking error. And $K(\tilde{\chi}(x, t))=\left[K_{1}(\tilde{\chi}(x, t))^{T}, \ldots, K_{p}(\tilde{\chi}(x, t))^{T}\right]^{T} \in \mathfrak{R}^{n p}$ is the nonlinear function of error states, and is specified at $p$ locations in $\Omega$, so that the tracking error $\tilde{\chi}(x, t)$ can be reduced to below an acceptable level despite the effects of random intrinsic parameter fluctuations and environmental disturbances.

The bounded environmental disturbance $d(x, t)$ is uncertain and unpredictable, and the bounded reference input signal $r(x, t)$ can be arbitrarily assigned by users and is thus unavailable to the designer beforehand. The robust reference tracking control is therefore designed so that the spatiotemporal effects of uncertainties $d(x, t)$ and $r(x, t)$ on the tracking error $\tilde{\chi}(x, t)$ can be attenuated below a prescribed level $\rho$ as follows:

$$
\frac{E \int_{0}^{t_{f}} \int_{\Omega}\left(\tilde{\chi}(x, t)^{T} \tilde{Q} \tilde{\chi}(x, t)\right) d x d t}{E \int_{0}^{t_{f}} \int_{\Omega}\left(v(x, t)^{T} v(x, t)\right) d x d t} \leq \rho^{2}
$$

which can be represented as:

$$
E \int_{0}^{t_{f}} \int_{\Omega}\left(\tilde{\chi}(x, t)^{T} Q \tilde{\chi}(x, t)\right) d x d t \leq \rho^{2} E \int_{0}^{t_{f}} \int_{\Omega}\left(v(x, t)^{T} v(x, t)\right) d x d t
$$

where $Q$ is the positive definite weighting matrix and $v(x, t)=$ $\left[r(x, t)^{T}, d(x, t)^{T}\right]^{T}$ is considered a vector of external disturbances. If the effect of the initial value $\tilde{\chi}(x, 0)$ is considered, i.e. $\tilde{\chi}(x, 0) \neq 0$, then we get:

$$
\begin{aligned}
& E \int_{0}^{t_{f}} \int_{\Omega}\left(\tilde{\chi}(x, t)^{T} \tilde{Q} \tilde{\chi}(x, t)\right) d x d t \leq E \int_{\Omega} V(\tilde{\chi}(x, 0)) d x+\rho^{2} E \\
& \int_{0}^{t_{f}} \int_{\Omega}\left(v(x, t)^{T} v(x, t)\right) d x d t
\end{aligned}
$$

for some positive Lyapunov function $V(\bullet)>0$. The inequality in (7) or (8) is the two-dimensional spatiotemporal robust $\mathrm{H} \infty$ tracking control performance. In other words, the effect of all possible disturbances on the tracking error with respect to average energy over the total space $\Omega$ must be attenuated below a prescribed level $\rho$.

Remark 1: To develop the optimal robust tracking control, the above two-dimensional robust $\mathrm{H} \infty$ tracking control problem must be solved. Then the upper bound $\rho^{2}$ can be minimised to derive the real optimal robust tracking control for the ecosystem, i.e. to minimise the tracking error under all possible disturbances over the spatiotemporal dimain. An optimal attenuation level $\rho_{0}$ can thus be obtained by solving the following constrained optimisation via the robust reference tracking control design $u(x, t)$ in (6):

$\rho_{0}=\min _{u(x, t)} \rho$

subject to (7) or (8)

To solve this problem, an augmented system that combines the dynamics of the ecosystem in (4) and the reference model in (5) is proposed as follows:

$$
d \bar{\chi}(x, t)=(F(\bar{\chi}(x, t))+E v(x, t)) d t+F_{\omega}(\bar{\chi}(x, t)) d \omega(x, t)
$$

where

$$
\bar{\chi}(x, t)=\left[\begin{array}{c}
\chi_{r}(x, t) \\
\chi(x, t)
\end{array}\right], \quad E=\left[\begin{array}{cc}
I_{n} & 0 \\
0 & I_{n}
\end{array}\right], \quad v(x, t)=\left[\begin{array}{l}
r(x, t) \\
d(x, t)
\end{array}\right],
$$




$$
\begin{aligned}
& F(\bar{\chi}(x, t))=\left[\begin{array}{l}
\left(A_{r} \chi_{r}(x, t)+D_{r} \nabla^{2} \chi_{r}(x, t)+V_{r} \nabla \chi_{r}(x, t)\right) \\
\left(f(\chi(x, t))+D \nabla^{2} \chi(x, t)+V \nabla \chi(x, t)+g(x) u(x, t)\right)
\end{array}\right], \\
& F_{\omega}(\bar{\chi}(x, t))=\left[\begin{array}{c}
0 \\
f_{\omega}(\chi(x, t))
\end{array}\right] .
\end{aligned}
$$

Thus, robust $\mathrm{H} \infty$ tracking control performance in (7) or (8) can be represented by:

$$
\frac{E \int_{0}^{t_{f}} \int_{\Omega}\left(\bar{\chi}(x, t)^{T} Q \bar{\chi}(x, t)\right) d x d t}{E \int_{0}^{t_{f}} \int_{\Omega}\left(v(x, t)^{T} v(x, t)\right) d x d t} \leq \rho^{2}
$$

or:

$$
E \int_{0}^{t_{f}} \int_{\Omega}\left(\bar{\chi}(x, t)^{T} Q \bar{\chi}(x, t)\right) d x d t \leq \rho^{2} E \int_{0}^{t_{f}} \int_{\Omega}\left(v(x, t)^{T} v(x, t)\right) d x d t
$$

where

$$
Q=\left[\begin{array}{cc}
\tilde{Q} & -\tilde{Q} \\
-\tilde{Q} & \tilde{Q}
\end{array}\right]
$$

If the effect of the initial value $\bar{\chi}(x, 0)$ is considered, the robust $\mathrm{H} \infty$ tracking control performance in (13) can be represented by:

$$
\begin{aligned}
& E \int_{0}^{t_{f}} \int_{\Omega}\left(\bar{\chi}(x, t)^{T} Q \bar{\chi}(x, t)\right) d x d t \leq E \int_{\Omega} V(\bar{\chi}(x, 0)) d x+\rho^{2} E \\
& \int_{0}^{t_{f}} \int_{\Omega}\left(v(x, t)^{T} v(x, t)\right) d x d t
\end{aligned}
$$

This leads to the following result for the robust reference tracking control strategy (6) for nonlinear stochastic bioticabiotic ecosystems.

Theorem 1: For the augmented system (11), with a prescribed disturbance attenuation level $\rho$ in (13) or (14), if there exists a robust reference tracking control strategy $u(x, t)$ in (6), such that the following HJII holds for $V(\bar{\chi}(x, t))>0$ :

$$
\int_{\Omega}\left(\begin{array}{l}
\bar{\chi}(x, t)^{T} Q \bar{\chi}(x, t)+\left(\frac{\partial V(\bar{\chi}(x, t))}{\partial \bar{\chi}(x, t)}\right)^{T} F(\bar{\chi}(x, t))+ \\
\frac{1}{4 \rho^{2}}\left(\frac{\partial V(\bar{\chi}(x, t))}{\partial \bar{\chi}(x, t)}\right)^{T} E E^{T}\left(\frac{\partial V(\bar{\chi}(x, t))}{\partial \bar{\chi}(x, t)}\right)+ \\
\frac{1}{2} F_{\omega}(\bar{\chi}(x, t))^{T}\left(\frac{\partial^{2} V(\bar{\chi}(x, t))}{\partial \bar{\chi}^{2}(x, t)}\right) F_{\omega}(\bar{\chi}(x, t))
\end{array}\right) d x<0
$$

then (i) the robust $\mathrm{H} \infty$ tracking performance in (13) or (14) is guaranteed by the robust reference tracking control strategy in (6); and (ii) if the ecosystem is free of environmental disturbance, $\chi(x, t)$ will asymptotically track the reference $\chi_{r}(x, t)$ in probability.

Proof: See Appendix A.

\section{Robust Fuzzy Tracking Control Design Approach}

To achieve the robust $\mathrm{H} \infty$ tracking control performance in (14), it would be necessary to solve a very complicated HJII in (15), which is difficult to do analytically or numerically. Even if we solve either, it is not easy to estimate $\chi(x, t)$ and $\chi_{r}(x, t)$ for the whole space $\Omega$ so that we can implement the robust reference tracking control strategy $u(x, t)$. In this situation, the following control design is proposed in order to simplify the robust reference tracking control design for nonlinear stochastic biotic-abiotic ecosystems. It uses a fuzzy interpolation scheme to overcome the nonlinearity and a finite differrence scheme to overcome the differential operators $\nabla$ and $\nabla^{2}$.

The T-S fuzzy model is described by fuzzy "If-then" rules and used here to solve the HJII in (15). The $i$ th rule of the T-S fuzzy model for the ecosystem in (4) is proposed as follows (Chen and Chang, 2009; Chen et al., 2012):

Rule $i$ :If $z_{1}(x, t)$ is $F_{i 1}, \ldots, z_{l}(x, t)$ is $F_{i l}$,

$$
\begin{aligned}
& \text { then } d \chi(x, t)=\left\{\left[A_{i} \chi(x, t)+D \nabla^{2} \chi(x, t)+V \nabla \chi(x, t)+\right.\right. \\
& \left.g(x) u(x, t)+d(x, t)] d t+A_{\omega i} \chi(x, t) d \omega(x, t)\right\}, i=1,2, \ldots, L
\end{aligned}
$$

where $A_{i} \in \mathfrak{R}^{n \times n}$ and $A_{\omega i} \in \mathfrak{R}^{n \times n}$ are the local linearised system matrices; $z_{i}(x, t)$ is the premise variable; $F_{i s}, s=1,2, \ldots, m$, is the fuzzy set and $m \leq n$ is the number of premise variables. If all state variables in $\chi(x, t)$ are used as premise variables then $m=n$. Following the "If-then" rules in (16), the overall fuzzy stochastic PDEs system for the ecosystem in (3) can be represented as follows (Chen et al., 1999; Tseng et al., 2001; Chen and Chang, 2009):

$$
\begin{aligned}
& d \chi(x, t)=\sum_{i=1}^{L} \mu_{i}(z(x, t))\left\{\left[A_{i} \chi(x, t)+D \nabla^{2} \chi(x, t)+V \nabla \chi(x, t)+\right.\right. \\
& g(x) u(x, t)+d(x, t)+\varepsilon(x, t)] d t+A_{\omega i} \chi(x, t) d \omega(x, t)+ \\
& \left.\varepsilon_{\omega}(x, t) d \omega(x, t)\right\}
\end{aligned}
$$

where the fuzzy interpolation bases $\mu_{i}(z(x, t))=\prod_{s=1}^{m} F_{i s}(z(x$, $t)) / \Sigma_{i=1}^{L} \Pi_{s=1}^{m} F_{i s}(z(x, t))$ for $i=1,2, \ldots, L, z(x, t)=\left[z_{1}(x, t), \ldots\right.$, $\left.z_{m}(x, t)\right]$, and $F_{i s}(z(x, t))$ are the grade or membership functions of $z(x, t)$ in $F_{i s}$. We assume $\prod_{s=1}^{m} F_{i s}(z(x, t)) \geq 0$ and $\sum_{i=1}^{L} \Pi_{s=1}^{m}$ $F_{i s}(z(x, t))>0$. Therefore, we get fuzzy bases $\mu_{i}(z(x, t)) \geq 0$ and the total sum of fuzzy bases $\Sigma_{i=1}^{L} \mu_{i}(z(x, t))=1$. The fuzzy approximation errors are defined as:

$\varepsilon(x, t)=f(\chi(x, t))-\sum_{i=1}^{L} \mu_{i}(z(x, t)) A_{i} \chi(x, t) ;$ 
$\varepsilon_{\omega}(x, t)=f_{\omega}(\chi(x, t))-\sum_{i=1}^{L} \mu_{i}(z(x, t)) A_{\omega i} \chi(x, t)$

Remark 2: In (17), applying the T-S fuzzy approach (Takagi and Sugeno, 1985) and the interpolation of $L$ local linear systems to approximate a nonlinear system, the effects of fuzzy approximation errors $\varepsilon(x, t)$ and $\varepsilon_{\omega}(x, t)$ are usually omitted for concise representation. The fuzzy approximation error is not only dependent on the complexity of the nonlinear system but also on the number of fuzzy rules $L$, i.e. $\varepsilon(x, t)$ and $\varepsilon_{\sigma}(x, t)$ decrease as $L$ increases. For a more precise approximation, the influence of the fuzzy approximation errors in (18) can be incorporated in the design of the robust reference tracking control, or it can be merged with the external disturbance $d(x, t)$, whose effect could be attenuated by the proposed robust reference tracking strategy. The details of how to deal with the effect of fuzzy approximation errors can be found in our previous studies (Tseng et al., 2001; Chen et al., 2009; Chang and Chen, 2010). In this study, we estimated the fuzzy approximation errors based on the following assumption:

Assumption 1 (Chen and Chang, 2009): If the functions $f(\chi(x, t))$ and $f_{\omega}\left(\chi(x, t)\right.$ are defined for a compact set $U \subset \mathfrak{R}^{n}$, then the fuzzy approximation errors would be bounded, i.e. $\|\varepsilon(x, t)\|^{2} \leq \sigma^{2}\|\chi(x, t)\|^{2}$ and $\left\|\varepsilon_{\omega}(x, t)\right\|^{2} \leq \sigma_{\omega}^{2}\|\chi(x, t)\|^{2}$, where $\sigma$ and $\sigma_{\omega}$ are the positive scalar values that depend on the number of fuzzy rules $L$.

Using the same logic, the nonlinear robust reference tracking control strategy in (6) could be redesigned based on the following fuzzy control law:

$$
\begin{aligned}
& \text { Rule } j: \text { If } z_{1}(x, t) \text { is } F_{j 1}, \ldots z_{l}(x, t) \text { is } F_{j l}, \\
& \text { then } u(x, t)=K_{j}(x, t) \tilde{\chi}(x, t)=K_{j}(x, t)\left(\chi(x, t)-\chi_{r}(x, t)\right), \\
& j=1,2, \ldots, L
\end{aligned}
$$

where $K_{j}(x, t)=\left[K_{j}\left(x^{l}, t\right)^{T}, \ldots, K_{j}\left(x^{p}, t\right)^{T}\right]^{T} \in \mathfrak{R}^{n p \times n}$ is the linear control gain at $p$ locations.

After approximating the nonlinear function using the fuzzy interpolation scheme, we use a finite difference scheme to treat the partial differential operators $\nabla$ and $\nabla^{2}$ in the ecosystem. Finite difference schemes have been widely used to obtain numerical solutions for PDEs. The main advantages of the finite difference method are that it is easy to understand and to implement. Because discretization is often intuitive, it is an efficient scheme for some PDEs problems. To design a robust reference tracking control for the ecosystem using a limited set of controllers, we should take into account the effects of environmental disturbances, the available number of control devices and the practical space partition size. Here, we consider a typical grid mesh with a uniform mesh space $\Delta$ as shown in Figure 2. The state vector $\chi(x, t)$ at the grid node $x_{k, l}=$ $[k \Delta, l \Delta]^{T}$ is represented by $\chi_{k, l}(t) \in \mathfrak{R}^{n}$, i.e. $\left.\chi(x, t)\right|_{x=x k, l}=\chi_{k, l}(t)$, where $k=1, \ldots, N_{l}, l=1, \ldots, N_{2}$. To satisfy the Dirichlet boundary conditions (Evans et al., 2000), the values of $\chi_{k, l}(t)$ at boundaries are fixed, i.e., if $\chi(x, t)=0$ at $\partial \Omega$, we have $\chi_{k, l}(t)=$ 0 at $k=0, N_{1}+1$ or $l=0, N_{2}+1$. The spatial state vector $\chi(t)$ $\in \mathfrak{R}^{n N}$ for state vectors at all grid nodes in the spatial domain $\Omega$ is defined as follows:

$$
\begin{aligned}
& \chi(t)=\left[\chi_{1,1}(t)^{T} \ldots \chi_{k, 1}(t)^{T} \ldots \chi_{N_{1}, 1}(t)^{T} \ldots \chi_{k, l}(t)^{T} \ldots \chi_{1, N_{2}}(t)^{T} \ldots\right. \\
& \left.\chi_{k, N_{2}}(t)^{T} \ldots \chi_{N_{1}, N_{2}}(t)^{T}\right]^{T}
\end{aligned}
$$

where $N \equiv N_{l}+N_{2}$. To simplify the index of the states $\chi_{k, l}(t)$ in the spatial state vector $\chi(t)$, we denote $\chi_{j}(t) \equiv \chi_{k, l}(t)$, where $j=(l-1) N_{l}+k$. We use the two-dimensional index $(k, l)$ to express the finite difference schemes of the two-index system, while the one-dimensional index $j$ is used in the equivalent one-index system. These two-dimensional indexing systems coincide in the one-dimensional case. Therefore, the two-dimensional index in (20) can be reduced to a one-dimensional index as follows:

$$
\begin{aligned}
& \chi(t)=\left[\chi_{1}(t)^{T} \ldots \chi_{k}(t)^{T} \ldots \chi_{N_{1}}(t)^{T} \ldots \chi_{j}(t)^{T} \ldots \chi_{N-N_{1}+1}(t)^{T} \ldots\right. \\
& \left.\chi_{N-N_{1}+k}(t)^{T} \ldots \chi_{N}(t)^{T}\right]^{T}
\end{aligned}
$$

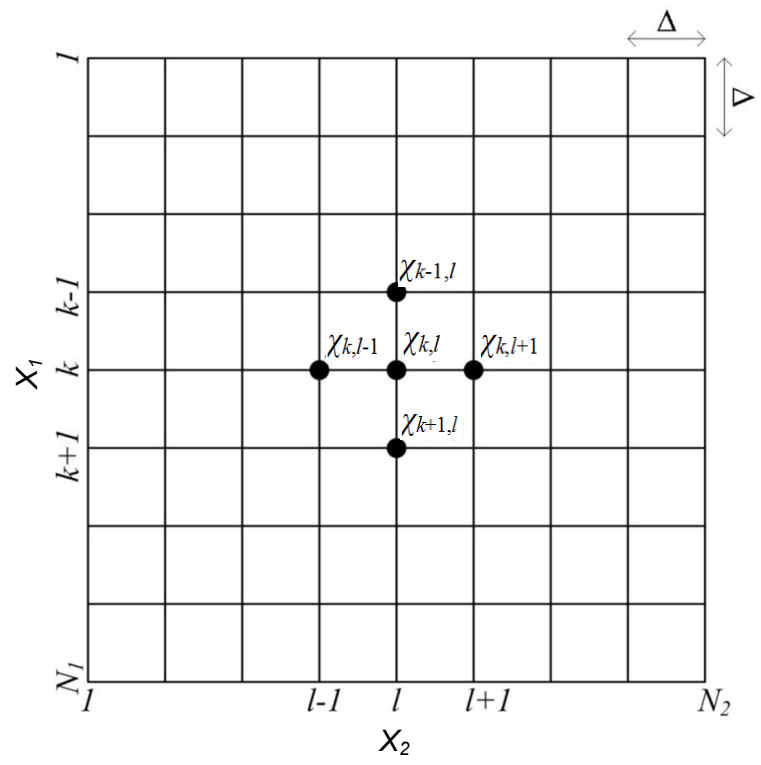

Figure 2. Finite difference grids $(\bullet)$ for two dimensional variables $X_{1}$ and $X_{2}$.

Remark 3: To satisfy the Neumann boundary conditions (Evans et al., 2000), i.e. $\partial \chi(x, t) / \partial x=0$ on $\partial \Omega$, the boundary is given as $\partial \chi(x, t) /\left.\partial x\right|_{x=x k, l}=0$ at $k=0, \ldots, N_{1}+1$ or $l=0, \ldots$, $N_{2}+1$. Therefore, if the grid nodes at boundaries are also considered in the spatial state vector $\chi(t) \in \mathfrak{R}^{n N}$, then $\chi(t)$ in (20) should be modified as follows:

$$
\begin{aligned}
& \chi(t)=\left[\chi_{0,0}(t)^{T} \ldots \chi_{k, 0}(t)^{T} \ldots \chi_{N_{1}+1,0}(t)^{T} \ldots \chi_{k, l}(t)^{T} \ldots \chi_{0, N_{2}+1}(t)^{T} \ldots\right. \\
& \left.\chi_{k, N_{2}+1}(t)^{T} \ldots \chi_{N_{1}+1, N_{2}+1}(t)^{T}\right]^{T}
\end{aligned}
$$

where $N \equiv\left(N_{1}+2\right)+\left(N_{2}+2\right)$. Similarly, if we let $j=l\left(N_{1}+2\right)$ $+(k+1)$, the two-dimensional index in (22) can also be redu- 
ced to a one-dimensional index as follows:

$$
\begin{aligned}
& \chi(t)=\left[\chi_{1}(t)^{T} \ldots \chi_{k+1}(t)^{T} \ldots \chi_{N_{1}+2}(t)^{T} \ldots \chi_{j}(t)^{T} \ldots \chi_{\left(N-N_{1}-2\right)+1}(t)^{T} \ldots\right. \\
& \left.\chi_{\left(N-N_{1}-2\right)+k}(t)^{T} \ldots \chi_{N}(t)^{T}\right]^{T}
\end{aligned}
$$

From (17), the fuzzy finite difference model of $\chi_{k, l}(t)$ at the grid node $x_{k, l}=[k \Delta, l \Delta]^{T}$ could be represented by $\chi_{j}(t)$ as follows:

$$
\begin{aligned}
& d \chi_{j}(t)=\sum_{i=1}^{L} \mu_{i}\left(z_{j}(t)\right)\left\{\left[A_{i} \chi_{j}(t)+D \nabla_{j}^{2} \chi(t)+V \nabla_{j} \chi(t)+B_{j} u(t)+\right.\right. \\
& \left.\left.d_{j}(t)+\varepsilon_{j}(t)+O_{j}\left(\Delta^{2}\right)\right] d t+A_{\omega i} \chi_{j}(t) d \omega_{j}(t)+\varepsilon_{\omega}(t) d \omega_{j}(t)\right\}
\end{aligned}
$$

where the influence matrix $B_{j}=\left[B_{x l}, \ldots, B_{x p}\right] \in \mathfrak{R}^{n \times n p}$ is defined as $B_{x i}=I_{n}$ for $x_{j}=x^{i}$ if a control device is located at $x_{j}$, or $B_{x i}=$ $0_{n}$ for $x_{j} \neq x^{i}$; the control input $u(t)=\left[u(t)_{x l}{ }^{T}, \ldots, u(t)_{x p}\right]^{T} \in$ $\Re^{n p}$.We assume that for any grid node $j$, the system spatial dynamics of $\chi_{j}(t)$ have a uniform influence over a unit of grid space, and that the differential operators $\nabla$ and $\nabla^{2}$ defined in (2) can be approximated by the finite difference matrices $\nabla_{j} \in$ $\mathfrak{R}^{n \times n N}$ and $\nabla_{j}^{2} \in \mathfrak{R}^{n \times n N}$. We express the interactions of other grid nodes with node $j$ using the following central difference approximation (Evans et al., 2000; Strikwerda, 2004):

$$
\begin{aligned}
& \nabla_{j} \chi(t)=\nabla_{k, l} \chi(t)=\frac{1}{2 \Delta}\left(\chi_{k+1, l}(t)-\chi_{k-1, l}(t)\right)+\frac{1}{2 \Delta}\left(\chi_{k, l+1}(t)-\right. \\
& \left.\chi_{k, l-1}(t)\right)=\frac{1}{2 \Delta}\left[\begin{array}{lllllll}
0_{n, 1} \ldots & -I_{n, j-N_{1}} & 0_{2} \ldots & -I_{n, j-1} & 0_{n, j} & I_{n, j+1}
\end{array}\right. \\
& \left.\begin{array}{lllll}
0_{n} & \ldots & I_{n, j+N_{1}} & \ldots & 0_{n, N}
\end{array}\right] \chi(t) \\
& \nabla_{j}^{2} \chi(t)=\nabla_{k, l}^{2} \chi(t)=\frac{1}{\Delta^{2}}\left(\chi_{k+1, l}(t)+\chi_{k-1, l}(t)-2 \chi_{k, l}(t)\right)+\frac{1}{\Delta^{2}} \\
& \left(\chi_{k, l+1}(t)+\chi_{k, l-1}(t)-2 \chi_{k, l}(t)\right)=\frac{1}{\Delta^{2}}\left[0_{n, 1} \ldots \quad I_{n, j-N_{1}} \quad 0_{n} \ldots \quad I_{n, j-1}\right. \\
& \left.\begin{array}{lllllll}
-4 I_{n, j} & I_{n, j+1} & 0_{n} & \ldots & I_{n, j+N_{1}} & \ldots & 0_{n, N}
\end{array}\right] \chi(t)
\end{aligned}
$$

The remainder term $O_{j}\left(\Delta^{2}\right) \equiv O_{\nabla_{j}}\left(\Delta^{2}\right)+O_{\nabla_{2 j}}\left(\Delta^{2}\right)$ in (24) is called the local truncation error. For concise representation, the local truncation errors $O_{\nabla_{j}}\left(\Delta^{2}\right) \equiv V \nabla \chi\left(x_{j}, t\right)-V \nabla_{j} \chi(t)$ and

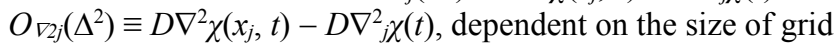
space $\Delta$, are usually omitted. This is possible because it has been proven that if the grid node points in the spatial domain are dense enough then the truncation error will be small enough to disregard (Yoo, 2000). Here however, we take the effects of these local truncation errors on tracking control performance into account. They can also be attenuated using our robust reference tracking control design.

After collecting all states $\chi_{j}(t)$ of the grid nodes in (24) into the spatial state vector $\chi(t)$ in (21), the fuzzy interpolation bases $\mu_{i}\left(z_{j}(t)\right)$ can be transformed into a matrix of diagonal fuzzy interpolation bases $\mu_{i}(z(t))=\operatorname{diag}\left(\mu_{i}\left(z_{1}(t)\right), \ldots\right.$, $\left.\mu_{i}\left(z_{N}(t)\right)\right) \in \mathfrak{R}^{N \times N}$. Using the Kronecker product, the represen- tation can be simplified and some properties of $\mu_{i}(z(t))$ can be obtained as follows.

Lemma 1: Properties of Kronecker product of fuzzy interpolation bases

1. $\mu_{i}(z(t)) \otimes A_{i}=\left(\mu_{i}(z(t)) \otimes I_{n}\right)\left(I_{N} \otimes A_{i}\right) \in \mathfrak{R}^{n N \times n N}$ where $A_{i} \in$ $\mathfrak{R}^{n \times n}$.

2. $\left(\mu_{i}(z(t)) \otimes I_{n}\right)\left(\mu_{j}(z(t)) \otimes I_{n}\right)=\left(\mu_{i}(z(t)) \mu_{j}(z(t)) \otimes I_{n}\right) \in$ $\Re^{n N \times n N}$.

$3 \sum_{i=1}^{L}\left(\mu_{i}(z(t))\right)=I_{N}, \sum_{i=1}^{L}\left(\mu_{i}(z(t)) \otimes I_{n}\right)=I_{n N}$ and $\sum_{i=1}^{L} \sum_{j=1}^{L}\left(\mu_{i}(z(t))\right.$

$\mu_{j}(z(t)) \otimes I_{n}=I_{n N}$.

The Kronecker product is a special case of the tensor product, so it is bilinear and associative. Properties 1 and Properties 2 are both derived from the so-called "Mixed-product property" of Kronecker product, because it mixes the matrix product and the Kronecker product, i.e., if $A, B, C$ and $D$ are matrices that can form the matrix products $A C$ and $B D$, then $(A \otimes B)(C \otimes D)=A C \otimes B D$, which is the fundamental properties for the Kronecker product. And Property 3 can be easily proven via the fuzzy fundamental property: $\Sigma_{i=1}^{L}\left(\mu_{i}\left(z_{j}(t)\right)\right)=$ 1 for $j=1,2, \ldots, N$. These properties of Kronecker product of fuzzy interpolation bases $\mu_{i}\left(z_{j}(t)\right)$ could simplify the system representation and tracking control design procedure of fuzzy reaction-diffusion-advection system in (24).

Using Lemma 1, the overall fuzzy finite difference models in (24) and (25) in $\Omega$ can be written as the following fuzzy spatial state space system to represent the ecosystem in (4):

$$
\begin{aligned}
& d \chi(t)=\sum_{i=1}^{L} \bar{\mu}_{i}(z(t))\left\{\left[\bar{A}_{i} \chi(t)+\bar{D} \bar{\nabla}^{2} \chi(t)+\bar{V} \bar{\nabla} \chi(t)+\bar{B} u(t)+\right.\right. \\
& \left.\left.d(t)+\varepsilon(t)+O\left(\Delta^{2}\right)\right] d t+\bar{A}_{\omega i} \chi(t) \circ d \omega(t)+\varepsilon_{\omega}(t) \circ d \omega(t)\right\}
\end{aligned}
$$

where $\bar{\mu}_{i}(z(t))=\left(\mu_{i}(z(t)) \otimes I_{n}\right) \in \mathfrak{R}^{n N \times n N}, \bar{A}_{i}=\left(I_{N} \otimes A_{i}\right) \in$ $\Re^{n N \times n N}, \bar{D}=\left(I_{N} \otimes D\right) \in \Re^{n N} \times n N, \bar{\nabla}^{2}=\left[\bar{\nabla}_{1}^{2 T}, \ldots, \bar{\nabla}_{N}^{2 T}\right]^{T} \in$ $\mathfrak{R}^{n N \times n N}, \bar{V}=\left(I_{N} \otimes V\right) \in \mathfrak{R}^{n N \times n N}, \bar{\nabla}=\left[\nabla_{1}^{T}, \ldots, \nabla_{N}^{T}\right]^{T} \in \mathfrak{R}^{n N \times n N}$, $\bar{B}=\left[B_{1}^{T}, \ldots, B_{N}^{T}\right]^{T} \in \mathfrak{R}^{n N \times n p}, d(t)=\left[d_{1}(t)^{T}, \ldots, d_{N}(t)^{T}\right]^{T} \in \Re^{n N}, \varepsilon(t)$ $=\left[\varepsilon_{1}(t)^{\mathrm{T}}, \ldots, \varepsilon_{N}(t)^{\mathrm{T}}\right] \in \mathfrak{R}^{n N}, O\left(\Delta^{2}\right)=\left[O_{1}\left(\Delta^{2}\right)^{T}, \ldots, O_{N}\left(\Delta^{2}\right)^{T}\right]^{T}$ $\in \mathfrak{R}^{n N}, \bar{A}_{\omega i}=\left(I_{N} \otimes A_{\omega i}\right) \in \mathfrak{R}^{n N \times n N}, \varepsilon_{\omega}(t)=\left[\varepsilon_{\omega 1}(t)^{\mathrm{T}}, \ldots, \varepsilon_{\omega N}(t)^{\mathrm{T}}\right]$ $\in \mathfrak{R}^{n N}$ and $\omega(t)=\left[\omega_{1}(t)^{T}, \ldots, \omega_{N}(t)^{T}\right]^{T} \in \mathfrak{R}^{n N}$.

Similarly, the reference model in (5) can be transformed into a linear spatial state space reference model as follows:

$$
d \chi_{r}(t)=\left(\bar{A}_{r} \chi_{r}(t)+\bar{D}_{r} \bar{\nabla}^{2} \chi_{r}(t)+\bar{V}_{r} \bar{\nabla} \chi_{r}(t)+r(t)+O_{r}\left(\Delta^{2}\right)\right) d t
$$

where $\chi_{r}(t)=\left[\chi_{r 1}(t)^{T}, \ldots, \chi_{r N}(t)^{T}\right]^{T} \in \mathfrak{R}^{n N}, \bar{A}_{r}=\left(I_{N} \otimes A_{r}\right) \in \mathfrak{R}^{n N}$, $\bar{V}_{r}=\left[V_{r, 1}^{T}, \ldots, V_{r, N}^{T}\right]^{T} \in \mathfrak{R}^{n N \times n N}, \bar{D}_{r}=\left[D_{r, 1}^{T}, \ldots, D_{r, N}^{T}\right]^{T} \in \mathfrak{R}^{n N \times n N}$, $r(t)=\left[r_{1}(t)^{\mathrm{T}}, \ldots, r_{N}(t)^{\mathrm{T}}\right] \in \mathfrak{R}^{n N}$ and $O_{r}\left(\Delta^{2}\right)=\left[O_{r 1}\left(\Delta^{2}\right)^{\mathrm{T}}, \ldots\right.$, $\left.O_{r N}\left(\Delta^{2}\right)^{\mathrm{T}}\right]^{\mathrm{T}} \in \mathfrak{R}^{n N}$. Based on the fuzzy spatial state space system in (26) and the linear spatial state space reference model in (27), the robust reference tracking control strategy in (6) 
or the overall robust fuzzy tracking control designed according to fuzzy control law in (19) can be represented by the following robust fuzzy tracking control strategy to simplify the design procedure:

$u(t)=\sum_{i=1}^{L} \sum_{j=1}^{N} \mu_{i}\left(z_{j}(t)\right) K_{i, j}\left(\chi_{j}(t)-\chi_{r, j}(t)\right)=\sum_{i=1}^{L} \bar{K}_{i} \bar{\mu}_{i}(z(t))(\chi(t)-$ $\left.\chi_{r}(t)\right)$

where $\bar{K}_{i} \equiv\left[K_{i, 1}, \ldots, K_{i, j}, \ldots, K_{i, N}\right] \in \mathfrak{R}^{n p \times n N}$ is the linear control gain to be designed, i.e. only the $p$ locations of $j=1,2, \ldots$, $N$ are selected to locate controllers.

By substituting the design of the fuzzy tracking controller in (28) into the fuzzy spatial state space system in (26) and combining with the linear spatial state space reference model in (27), the augmented stochastic system in (11) could be modified to the following fuzzy augmented system:

$$
\begin{aligned}
& d \bar{\chi}(t)=\sum_{i=1}^{L} \sum_{j=1}^{L} \overline{\bar{\mu}}_{i}(z(t)) \overline{\bar{\mu}}_{j}(z(t))\left(\left(\bar{A}_{i j} \bar{\chi}(t)+\bar{E} \bar{v}(t)+\bar{\varepsilon}(t)\right) d t+\right. \\
& \left.\left(\bar{A}_{\bar{\omega} i} \bar{\chi}(t)+\bar{\varepsilon}_{\bar{\omega}}(t)\right) \circ d \bar{\omega}(t)\right)
\end{aligned}
$$

where $\overline{\bar{\mu}}_{i}(z(t))=\left(I_{2} \otimes \bar{\mu}_{i}(z(t))\right), \bar{\chi}(t)=\left[\chi_{r}(t)^{\mathrm{T}} \quad \chi(t)^{\mathrm{T}}\right]^{\mathrm{T}}, \bar{v}(t)=$ $\left[\begin{array}{ll}r(t)^{\mathrm{T}} O_{r}\left(\Delta^{2}\right)^{\mathrm{T}} d(t)^{\mathrm{T}} O\left(\Delta^{2}\right)^{\mathrm{T}}\end{array}\right]^{\mathrm{T}}, \bar{\varepsilon}(t)=\left[\begin{array}{ll}0 & \varepsilon(t)^{\mathrm{T}}\end{array}\right]^{\mathrm{T}}$, and $\bar{\varepsilon}_{\bar{\omega}}(t)=[0$ $\left.\varepsilon_{\omega}(t)^{\mathrm{T}}\right]^{\mathrm{T}}, \bar{\omega}(t)=\left[\begin{array}{ll}0 & \omega(t)^{\mathrm{T}}\end{array}\right]^{\mathrm{T}}$. The other notations are defined as follows:

$$
\begin{aligned}
& \bar{A}_{i j}=\left[\begin{array}{cc}
A_{11} & 0 \\
A_{21} & A_{22}
\end{array}\right]=\left[\begin{array}{c|c}
\bar{A}_{r}+\bar{D}_{r} \bar{\nabla}^{2}+\bar{V}_{r} \bar{\nabla} & 0 \\
\hline-\bar{B}_{j} & \bar{A}_{i}+\bar{D} \bar{\nabla}^{2}+\bar{V} \bar{\nabla}+\bar{B}_{j}
\end{array}\right], \\
& \bar{E}=\left[\begin{array}{cc}
E_{11} & 0 \\
0 & E_{22}
\end{array}\right]=\left[\begin{array}{cc|cc}
I_{n N} & I_{n N} & 0 & 0 \\
\hline 0 & 0 & I_{n N} & I_{n N}
\end{array}\right], \\
& \bar{A}_{\bar{\omega} i}=\left[\begin{array}{cc}
0 & 0 \\
0 & A_{\bar{\omega} 22}
\end{array}\right]=\left[\begin{array}{c|c}
0 & 0 \\
\hline 0 & \bar{A}_{\omega i}
\end{array}\right]
\end{aligned}
$$

Because the spatial state vector is used to represent $\chi(x, t)$ at all grid points, the spatiotemporal forms of $E \int_{0}^{t_{f}} \int_{\Omega}\left(\bar{\chi}(x, t)^{T}\right.$ $Q \bar{\chi}(x, t)) d x d t$ and $E \int_{0}^{t_{f}} \int_{\Omega}\left(v(x, t)^{T} v(x, t)\right) d x d t$ in the two-dimensional $\mathrm{H} \infty$ tracking control performance in (12) or (13) can be transformed to the temporal forms $E \int_{0}^{t_{f}}\left(\bar{\chi}(t)^{T} \bar{Q} \bar{\chi}(t)\right) \Delta^{2} d t$ and $E \int_{0}^{t_{f}}\left(\bar{v}(t)^{T} \bar{v}(t)\right) \Delta^{2} d t$, respectively. In this situation, the Lyapunov function $E \int_{\Omega} V(\bar{\chi}(x, 0)) d x$ in (14) can also be transformed to $E V(\bar{\chi}(0)) \Delta^{2}$, where $V(\bar{\chi}(t))=\sum_{j=1}^{N} V\left(\bar{\chi}_{j}(t)\right)$. The two-dimensional $\mathrm{H} \infty$ tracking control performance in (12) is therefore equivalent to the following inequality:

$$
\frac{E \int_{0}^{t_{f}}\left(\bar{\chi}(t)^{T} \bar{Q} \bar{\chi}(t)\right) \Delta^{2} d t}{E \int_{0}^{t_{f}}\left(\bar{v}(t)^{T} \bar{v}(t)\right) \Delta^{2} d t}=\frac{E \int_{0}^{t_{f}}\left(\bar{\chi}(t)^{T} \bar{Q} \bar{\chi}(t)\right) d t}{E \int_{0}^{t_{f}}\left(\bar{v}(t)^{T} \bar{v}(t)\right) d t} \leq \rho^{2}
$$

where the weighting matrices $\bar{Q}=Q \otimes I_{N}$. If the initial condi- tion is set as $\bar{\chi}(0) \neq 0$, i.e. there exists some positive function $V(\bar{\chi}(0))$, then the Hळ reference tracking performance in (14) can also be represented by the following inequality:

$$
E \int_{0}^{t_{f}}\left(\bar{\chi}(t)^{T} \bar{Q} \bar{\chi}(t)\right) d t \leq E V(\bar{\chi}(0))+\rho^{2} E \int_{0}^{t_{f}}\left(\bar{v}(t)^{T} \bar{v}(t)\right) d t
$$

To solve the $H \infty$ reference tracking problem in (31), let us choose a Lyapunov function:

$V(\bar{\chi}(t))=\bar{\chi}(t)^{T} \bar{P} \bar{\chi}(t)>0$

where $\bar{P}=\bar{P}^{T}>0$. Because the Lyapunov function is of the form $V(\bar{\chi}(t))=\sum_{j=1}^{N} V\left(\bar{\chi}_{j}(t)\right)$ in the finite difference system, we can define it for the augmented system of the $j$-th fuzzy finite difference system in (24) and the $j$-th reference finite difference system as follows:

$$
\begin{aligned}
& V\left(\bar{\chi}_{j}(t)\right)=V\left(\chi_{r, j}(t), \chi_{j}(t)\right)=\left[\begin{array}{c}
\chi_{r, j}(t) \\
\chi_{j}(t)
\end{array}\right]^{T}\left[\begin{array}{cc}
P_{11, j} & P_{12, j} \\
P_{12, j} & P_{11, j}
\end{array}\right]\left[\begin{array}{c}
\chi_{r, j}(t) \\
\chi_{j}(t)
\end{array}\right], \\
& j=1, \ldots, N
\end{aligned}
$$

The following form for the matrix $\bar{P}$ can therefore easily be obtained for the Lyapunov function in (32) as:

$\bar{P}=\left[\begin{array}{ll}P_{11} & P_{12} \\ P_{12} & P_{11}\end{array}\right]>0$

where $P_{11}=\operatorname{diag}\left(P_{11,1}, \ldots, P_{11, N}\right) \in \mathfrak{R}^{n N \times n N}$ and $P_{12}=\operatorname{diag}$ $\left(P_{12,1}, \ldots, P_{12, N}\right) \in \mathfrak{R}^{n N \times n N}$. If there exist some Lyapunov functions such as (32), then based on the positive matrix $\bar{P}$ in (33), we can obtain the following sufficient conditions for the $\mathrm{H} \infty$ tracking performance in (30) or (31).

Theorem 2: For the augmented system (29), with a prescribed disturbance attenuation level $\rho$ in (30), if there exists a symmetric, positive definite matrix $\bar{P}$ and the control gains $\bar{K}_{i}$ for fuzzy rules $i, j=1,2, \ldots, L$, such that the following matrix inequalities hold:

$$
\left[\begin{array}{ccc}
\bar{A}_{i j}^{T} \bar{P}+\bar{P}_{i j}+\bar{Q}+\bar{\Sigma}^{T} \bar{\Sigma}+2\left(\bar{A}_{\bar{\omega} i}+\bar{\Sigma}_{\bar{\omega}}\right)^{T} \bar{P}\left(\bar{A}_{\bar{\omega} j}+\bar{\Sigma}_{\bar{\omega}}\right) & \bar{P} \bar{E} & \bar{\Sigma}^{T} \\
\bar{E}^{T} \bar{P} & -\rho^{2} I & 0 \\
\bar{\Sigma} & 0 & -I
\end{array}\right]
$$

$<0, i, j=1,2, \ldots, L$

where $\bar{\Sigma}=\left[\begin{array}{ll}0 & \delta I\end{array}\right], \bar{\Sigma}_{\bar{\omega}}=\operatorname{diag}\left(0, \delta_{\omega} I\right)$, then (i) the Hळ tracking control performance for (31) with disturbance attenuation level $\rho$ is guaranteed by the robust fuzzy tracking control strategy in (28); and (ii) if the external disturbance $\bar{v}(t) \equiv 0$, then $\chi(t)$ will asymptotically track $\chi_{r}(t)$ in probability.

\section{Proof: See Appendix B.}

We see from $\bar{A}_{i j}$, defined for the augmented system in (29), 
that the control gains $\bar{K}$ would couple with the Lyapunov ma$\operatorname{trix} \bar{P}$ in the matrix inequality (34) when solving such tracking control problems (Chen et al., 2000). In this situation, the matrix inequality in (34) will be a complex bilinear matrix inequality (BMI). Because the BMI problem is non-convex, some algorithms for solving local BMI solutions using the augmented Lagrangian method (Kocvara and Stingl, 2003) and the iteration method (Chen et al., 2000; Huang and Nguang, 2006; $\mathrm{Wu}$ and $\mathrm{Li}, 2008$ ) have been proposed. However, these algorithms are inefficient for solving BMI problems with multiple variables. To reduce the BMI problem in (34) to an LMI problem, let us define the following matrix:

$$
\bar{X}=\left[\begin{array}{ll}
X_{11} & X_{12} \\
X_{12} & X_{11}
\end{array}\right]=\left[\begin{array}{ll}
P_{11} & P_{12} \\
P_{12} & P_{11}
\end{array}\right]^{-1}
$$

The BMI problem in (34) can then be transformed, which results in the following LMI problem.

Theorem 3: For the augmented system (29), with a prescribed disturbance attenuation level $\rho$ in (30), if there existse a symmetric, positive definite matrix $\bar{X}$ and the control gains $\bar{K}_{i}$ for $i, j=1,2, \ldots, L$, such that the following LMIs hold:

$$
\left[\begin{array}{ccccc}
\bar{X}_{i j}^{T}+\bar{A}_{i j} \bar{X}+I & \bar{X} \bar{Q}_{1 / 2}^{T} & \bar{X}\left(\bar{A}_{\bar{\omega} j}+\bar{\Sigma}_{\bar{\omega}}\right)^{T} & \bar{E} & \bar{X} \bar{\Sigma}^{T} \\
\bar{Q}_{1 / 2} \bar{X} & -I & 0 & 0 & 0 \\
\left(\bar{A}_{\bar{\omega} i}+\bar{\Sigma}_{\bar{\omega}}\right) \bar{X} & 0 & -\frac{1}{2} \bar{X} & 0 & 0 \\
\bar{E}^{T} & 0 & 0 & -\rho^{2} I & 0 \\
\bar{\Sigma} \bar{X} & 0 & 0 & 0 & -I
\end{array}\right]<0, \quad i,
$$

$j=1,2, \ldots, L$.

where $\bar{Q}_{1 / 2}=\left[Q_{1 / 2}-Q_{1 / 2}\right] \otimes I_{N}$ and $Q=Q_{1 / 2}^{T} Q_{1 / 2}$, then (i) the H $\infty$ tracking performance in (31) with a disturbance attenuation level $\rho$ is guaranteed by the fuzzy reference tracking control strategy in (28); and (ii) $\chi(t)$ will asymptotically track $\chi_{r}(t)$ in probability if $\bar{v}(t) \equiv 0$.

\section{Proof: See Appendix C.}

Solving the above LMIs, the robust $\mathrm{H} \infty$ tracking control strategy for nonlinear stochastic biotic-abiotic ecosystems can be designed with a prescribed disturbance attenuation level $\rho$. The optimal disturbance attenuation design is to specify tracking control gains $\bar{K}_{j}$ in (28) such that $\rho$ is as small as possible. To achieve the optimal robust $\mathrm{H} \infty$ tracking control performance, we can solve the following constrained optimisation problem:

$$
\rho_{0}=\min _{\bar{K}_{j}, j=1, \ldots, L} \rho
$$

Subject to $\bar{X}>0, \tilde{Q}>0$ and the LMIs in (36).

The minimum disturbance attenuation level $\rho_{0}$ can be used to measure the optimal robust $\mathrm{H} \infty$ tracking control performance.
The design procedure for robust $\mathrm{H} \infty$ tracking controls for nonlinear stochastic biotic-abiotic ecosystems can therefore be summarised as follows.

\section{Design procedures:}

Step 1: Given the desired reference model in (5), generate the reference output $\chi_{r}(x, t)$ and a prescribed disturbance attenuation level $\rho$.

Step 2: Select the fuzzy membership functions and fuzzy rules to establish the fuzzy stochastic PDEs system in (17) to approximate the nonlinear stochastic PDEs system in (4).

Step 3: Assign values to grid spacing $\Delta$ and grid number $N$ to construct the fuzzy spatial state space model in (26) and (27), and determine the $p$ locations of controllers.

Step 4: Obtain the control gains $\bar{K}_{j}$ for $j=1,2, \ldots, L$ in (28) by solving the LMIs problem in (36) or solving (37) for the optimal robust $\mathrm{H} \infty$ tracking control performance.

Step 5: Construct a fuzzy reference tracking control strategy in (28) to control the ecosystem in (4) and track the desired reference model in (5).

\section{Computer Simulation and Results}

To evaluate the performance of the proposed robust fuzzy tracking control for nonlinear stochastic biotic-abiotic ecosystems, the following nonlinear biomass-nutrient ecosystem (Klausmeier, 1999; Tilman et al., 2002) with stochastic parametric fluctuation and environmental disturbance is given as an example:

$$
\left\{\begin{array}{l}
\frac{\partial m(x, t)}{\partial t}=\left\{(W-L m(x, t)-R m(x, t) b(x, t))+\left(W_{\omega}-L_{\omega} m(x, t)-\right.\right. \\
\left.R_{\omega} m(x, t) b(x, t)\right) n(x, t)+v_{1}\left(\frac{\partial m(x, t)}{\partial x_{1}}+\frac{\partial m(x, t)}{\partial x_{2}}\right)+d_{1}\left(\frac{\partial^{2} m(x, t)}{\partial x_{1}^{2}}\right. \\
\left.+\frac{\partial^{2} m(x, t)}{\partial x_{2}^{2}}\right)+d_{m}(x, t) \\
\frac{\partial b(x, t)}{\partial t}=\left\{\left(J m(x, t) b(x, t)-M \frac{b(x, t)}{1+b(x, t)}\right)+\left(J_{\omega} m(x, t) b(x, t)-\right.\right. \\
\left.M_{\omega} \frac{b(x, t)}{1+b(x, t)}\right) n(x, t)+d_{2}\left(\frac{\partial^{2} b(x, t)}{\partial x_{1}^{2}}+\frac{\partial^{2} b(x, t)}{\partial x_{2}^{2}}\right)+d_{b}(x, t)
\end{array}\right.
$$

In the above nonlinear stochastic PDEs system, $m(x, t)$ denotes mineral nutrient concentration; $b(x, t)$ denotes plant biomass density; $W$ denotes basic nutrient supply; $L$ denotes nutrient loss; $R$ denotes nutrient uptake by biomass; $J$ denotes biomass yield; and $M$ denotes biomass mortality. The stochastic parametric fluctuations can be separated into deterministic and random parts, where $W_{\omega}, L_{\omega}, R_{\omega}, J_{\omega}$ and $M_{\omega}$ are the standard deviations of parametric fluctuations, and $n(x, t)$ is standard white noise with zero mean and unit variance. The terms $d_{m}(x, t)$ and $d_{b}(x, t)$ denote environmental disturbances due to rainfall variability, droughts, pest outbreaks or infectious disea- 
ses. In this case, nutrient flows with speed $v_{1}$ and diffusion rate $d_{1}$, and biomass dispersal is modelled by a diffusion term with diffusion coefficient $d_{2}$.

For NRM, a control input $u(x, t)=\left[u_{m}(x, t) u_{b}(x, t)\right]^{T}$ is introduced to regulate the biomass-nutrient ecosystem in (38), where $u_{m}(x, t)$ denotes nutrient pumps or conduits to regulate nutrient supply, and $u_{b}(x, t)$ denotes chemical pesticides or herbivore exclosures to regulate biomass production. By using a set of control devices set up at $p$ points, the ecosystem in (38) can be represented as the following Ito stochastic system:

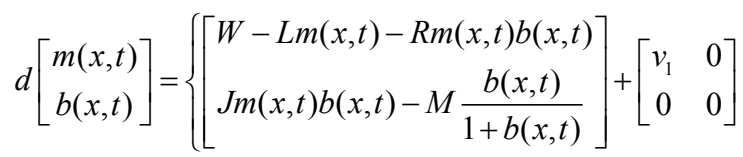

$\nabla\left[\begin{array}{c}m(x, t) \\ b(x, t)\end{array}\right]+\left[\begin{array}{cc}d_{1} & 0 \\ 0 & d_{2}\end{array}\right] \nabla^{2}\left[\begin{array}{c}m(x, t) \\ b(x, t)\end{array}\right]+\left[\left[\begin{array}{ll}1 & 0 \\ 0 & 1\end{array}\right]_{x^{1}}, \ldots,\left[\begin{array}{ll}1 & 0 \\ 0 & 1\end{array}\right]_{x^{p}}\right]$

$\left.\left[\left[\begin{array}{l}u_{m}\left(x^{1}, t\right) \\ u_{b}\left(x^{1}, t\right)\end{array}\right]^{T}, \ldots,\left[\begin{array}{l}u_{m}\left(x^{p}, t\right) \\ u_{b}\left(x^{p}, t\right)\end{array}\right]^{T}\right]^{T}+\left[\begin{array}{l}d_{m}(x, t) \\ d_{b}(x, t)\end{array}\right]\right\} d t+$

$\left[\begin{array}{l}W_{\omega}-L_{\omega} m(x, t)-R_{\omega} m(x, t) b(x, t) \\ J_{\omega} m(x, t) b(x, t)-M_{\omega} \frac{b(x, t)}{1+b(x, t)}\end{array}\right] d \omega(x, t)$

In this case, the values of system parameters are given in Table 2 (Tilman et al. 2002), $W_{\omega}=0.1 W, L_{\omega}=0.1 L, R_{\omega}=0.1 R$, $J_{\omega}=0.1 J$, and $M_{\omega}=0.1 M$. The initial value is given as $[m(x, 0)$ $b(x, 0)]^{T}=\left[\begin{array}{ll}4 & 10\end{array}\right]^{T}$ and the Dirichlet boundary condition is given as $[m(x, t) b(x, t)]^{T}=\left[\begin{array}{ll}0 & 0\end{array}\right]^{T}$ at boundary $\partial \Omega$.

To verify the performance of the robust fuzzy tracking control, we use a prescribed reference model based on human demand. The transient response and steady state of the reference model is specified by setting $A_{r}$ and $r(x, t)$, respectively, to determine the prescribed trajectory behaviour of the biomassnutrient ecosystem. In this case, to control the nonlinear biomass-nutrient ecosystem and let its spatial distribution appear a prescribed time dynamic, the following reference model is used:

$d\left[\begin{array}{c}m_{r}(x, t) \\ b_{r}(x, t)\end{array}\right]=\left(100 \times\left[\begin{array}{cc}-1 & 0 \\ 0 & -1\end{array}\right]\left[\begin{array}{c}m_{r}(x, t) \\ b_{r}(x, t)\end{array}\right]+\left[\begin{array}{ll}0 & 0 \\ 0 & 0\end{array}\right] \nabla\left[\begin{array}{c}m_{r}(x, t) \\ b_{r}(x, t)\end{array}\right]+\right.$

$\left.\left[\begin{array}{ll}0 & 0 \\ 0 & 1\end{array}\right] \nabla^{2}\left[\begin{array}{c}m_{r}(x, t) \\ b_{r}(x, t)\end{array}\right]+100 \times\left[\begin{array}{c}4+\sin (t) \\ 10+\cos (t)\end{array}\right]\right) d t$

In other words, by using the robust fuzzy tracking control, the trajectory of the nonlinear stochastic biomass-nutrient ecosystem can achieve a default spatial distribution according to the above prescribed reference model. In this case, the tracking controls $u(x, t)$ set up at $p$ points are also used to attenuate the effects of uncertainties on the tracking error $\chi(x, t)-\chi_{r}(x, t)$ below a prescribed attenuation level $\rho$, or to obtain the optimal robust $\mathrm{H} \infty$ tracking control performance $\rho_{0}$.

For the purpose of designing a robust reference tracking control using a set of $p$ controllers, the spatial domain $\Omega=[0$, $1] \times[0,1]$ is divided into $6 \times 6$ grid points with Dirichlet boundary conditions, so that the grid spacing $\Delta$ is 0.2 . The ecosystem can then be represented as a fuzzy spatial state space model using four T-S fuzzy rules and 36 finite difference grid points. The parameters $A_{i}$ and $A_{\omega i}$ can easily be identified using the fuzzy toolbox in Matlab, based on the triangular fuzzy membership function, which is shown as:

$$
\begin{aligned}
& A_{1}=\left[\begin{array}{rr}
-1.8500 & -0.3681 \\
6.9565 & 1.2624
\end{array}\right], A_{2}=\left[\begin{array}{rr}
-4.1557 & -0.3780 \\
20.8449 & 1.8135
\end{array}\right], A_{3}= \\
& {\left[\begin{array}{rr}
-1.8473 & -0.5871 \\
6.7578 & 2.6482
\end{array}\right], A_{4}=\left[\begin{array}{rr}
-4.0713 & -0.5547 \\
20.3887 & 2.9035
\end{array}\right]} \\
& A_{\omega 1}=\left[\begin{array}{rr}
-0.1850 & -0.0368 \\
0.6957 & 0.1262
\end{array}\right], A_{\omega 2}=\left[\begin{array}{rr}
-0.4156 & -0.0378 \\
2.0845 & 0.1814
\end{array}\right], A_{\omega 3}= \\
& {\left[\begin{array}{cc}
-0.1847 & -0.0587 \\
0.6758 & 0.2648
\end{array}\right], A_{\omega 4}=\left[\begin{array}{cc}
-0.4071 & -0.0555 \\
2.0389 & 0.2903
\end{array}\right]}
\end{aligned}
$$

We can calculate the bounds of the fuzzy approximation errors as $\sigma=0.3219$ and $\sigma_{\omega}=0.0322$, according to the definition in (18) and Assumption 1. By solving the LMIs-constrained problem in (36), we can calculate the control gains $\bar{K}_{j}$ for different distribution control strategies. To investigate the robust tracking performance of different control strategies, we use three examples of distribution control strategies (Figure 6). In Case 1, the all-points distribution control, the control devices were set up at all interior grid points. In Case 2, the gap-points distribution control, half the number of control devices was set up, and they were placed at non-adjacent interior grid points. In Case 3, the column-points distribution control, half the number of control devices was set up, and they were placed in non-adjacent columns of interior grid points. The three optimal $H \infty$ robust tracking control performance $\rho_{0}$ as shown in Figure 6 can thus be obtained by solving the optimal robust tracking control problem in (37) for each case. These control strategies utilising different arrangements and numbers of control devices may then be compared.

Table 2. Interpretation of Symbols and Parameter Values Used (Tilman et al., 2002)

\begin{tabular}{lll}
\hline Symbols & Interpretation & Value \\
\hline $\mathrm{L}$ & nutrient lost & 0.72 \\
$\mathrm{R}$ & nutrient uptake by biomass & 1 \\
$\mathrm{~J}$ & yield of biomass & 6.10 \\
$\mathrm{~W}$ & basic nutrient supply & 0.72 \\
$\mathrm{M}$ & biomass mortality & 5.14 \\
$\mathrm{~V} 1$ & nutrient flow & -1.315 \\
$\mathrm{~d}_{1}$ & nutrient dispersal & 1 \\
$\mathrm{~d}_{2}$ & biomass dispersal & 2 \\
\hline
\end{tabular}

The simulation results show that the ecosystem can be robustly controlled by the finite control devices to track a prescribed reference system dynamic (Figures 3 to 5) with an opti- 

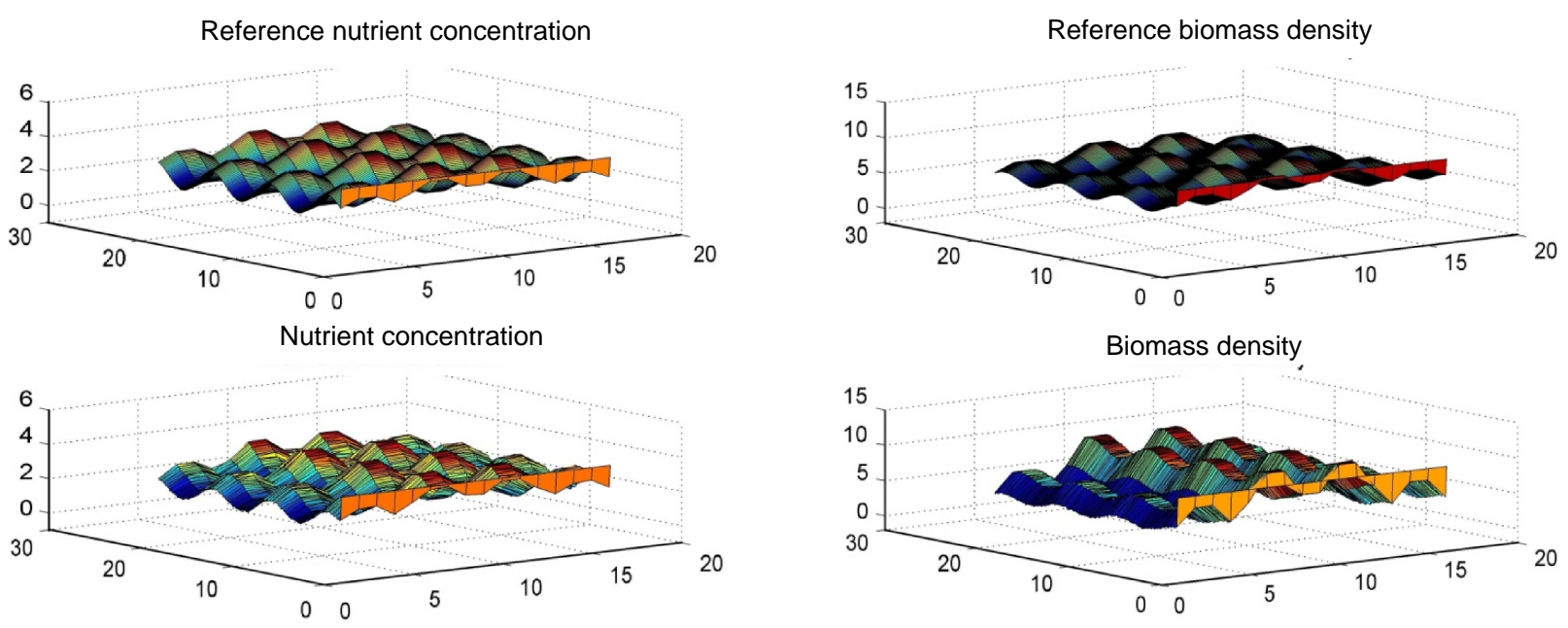

Tracking error of nutrient concentration

Tracking error of biomass density
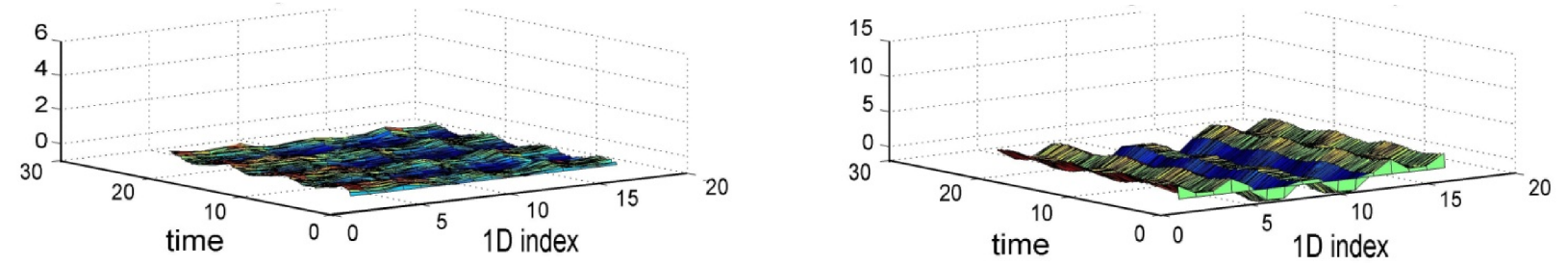

Figure 3. The spatiotemporal profiles of the optimal robust $\mathrm{H} \infty$ tracking control of the nonlinear stochastic nutrient-biomass system in (39) with control device distribution arrangement in Case 1 of Figure 6.

mal disturbance attenuation level $\rho_{0}$. This efficiently attenuates the effects of random parameter fluctuations, environmental noise and truncation errors. Managers can therefore manage natural resources in the field according to their needs using a set of controllers placed at a limited number of points, using the proposed tracking control strategy. Ecological patterns can also be adjusted as desired, regardless of the effects of advection, diffusion or external disturbances. The current distribution of natural resources, dictated by slope, plant tropism or animal taxis, random movement or environmental change, can therefore be overridden in this controlled ecosystem. The different distribution control strategies yielded the following robust tracking results to efficiently attenuate the effect of stochastic disturbances on the desired reference tracking: in Case 1, $\rho_{0}^{2}=$ $0.0153^{2}$, in Case 2, $\rho_{0}^{2}=0.0173^{2}$, and in Case 3, $\rho_{0}^{2}=0.0429^{2}$ (Figure 6). The optimal $\mathrm{H} \infty$ robust tracking control performance $\rho_{0}$ is therefore affected by both the number and arrangement of control devices. Under an acceptable tracking control performance, managers can control nutrient supply and biomass production via a set of control devices arranged according to the real constraints. These results are conservative, due to the conservative way in which we solved LMIs in the robust reference tracking control design, but the method nonetheless has many advantages, such as intuitive and easy implementation, practical use, and applicability to many different biophysical PDEs systems.

\section{Discussion and Conclusions}

In recent years, sustainable development has received in- creasing attention from scientific workers, governments and policy-makers around the world. Related studies are dedicated to preventing ecosystem degradation or managing agricultural production. In preventing ecosystem degradation, analyzing ecological data could help people access critical ecological information, e.g., wetland water quality, therefore people could introduce flow regulation to recharge the drying wetland for maintaining ecological balance, or change the land use pattern to reduce pollution for improving reservoir water quality (Chen et al., 2013; Recknagel, 2013; Yang and Chen, 2013). In managing agricultural production, a conservative planning scheme that could allow specific system violation has been proposed to solve the water quality management problem (Xu and Qin, 2013). Previous works have proposed helpful methods to regulate ecosystems, but none of them talking about regulating the ecosystem according to predetermined dynamic behaviours. With the population increasing, issues related to food security become increasingly severe especially in tropical developing countries. Thus managing the agricultural production for satisfying human demands (i.e., the projected human population) via efficient nutrient supply and pest control is important and urgent. In this study, we devote to developing the natural resource management for natural ecosystems and agricultural ecosystems via model reference tracking control design. Since the crop yields may be affected by individual differences and climate changes, managing the crop yield for achieving a desired amount needs to regulate a biomass-nutrient ecosystem under the influence of random intrinsic fluctuations and uncertain external disturbances. Because the statistical properties of environmental disturbances and artificial reference signals are 


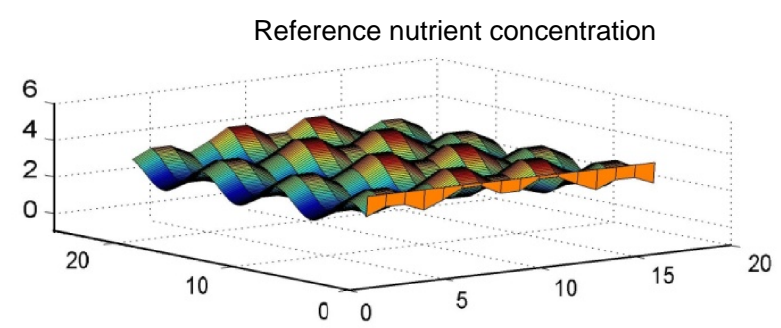

Nutrient concentration

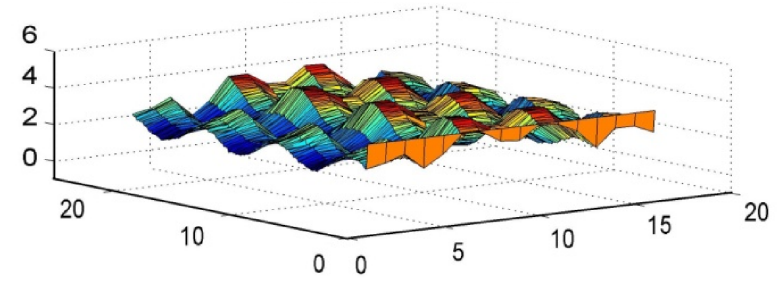

Tracking error of nutrient concentration

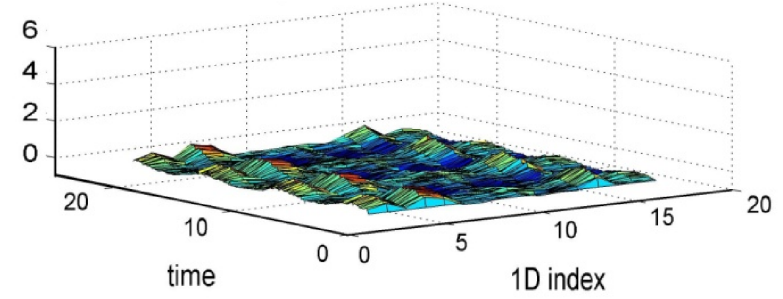

Reference biomass density

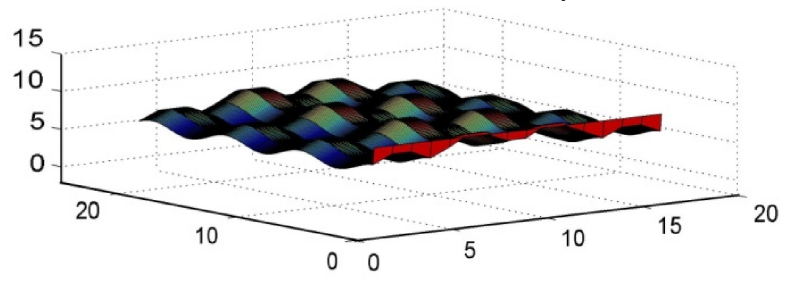

Biomass density

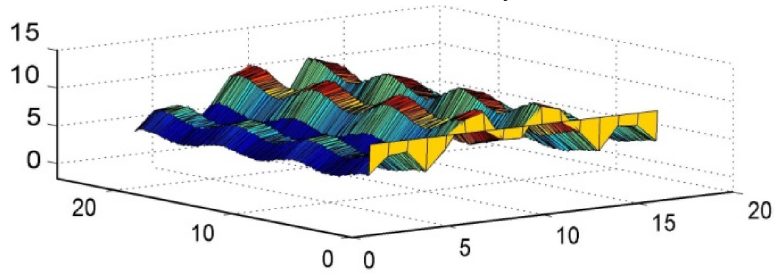

Tracking error of biomass density

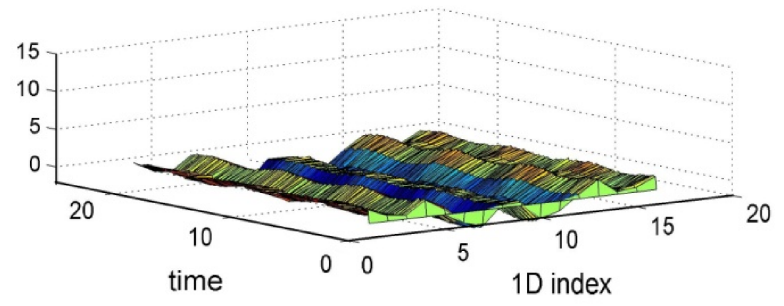

Figure 4. The spatiotemporal profiles of the optimal robust $\mathrm{H} \infty$ tracking control of the nonlinear stochastic nutrient-biomass system in (39) with control device distribution arrangement in Case 2 of Figure 6.

mostly unknown in reality, we propose a robust $\mathrm{H} \infty$ tracking control to ensure that the control strategy is effective and that the desired yield is achieved, irrespective of these intrinsic and external uncertainties.

Most analyses and manipulations of ecosystems are conducted in homogeneous conditions, either in highly artificial environments or in the field. However, in real ecosystems natural resources are always spatially and temporally heterogeneous. We develop a robust reference tracking control strategy for heterogeneously distributed natural resources (water or crops) that have additional complexities for shaping the spatiotemporal distribution, and attempt to eliminate the effects of random intrinsic fluctuation and uncertain external disturbance on reference tracking. To design the robust reference tracking control strategy of a nonlinear stochastic PDEs system, it is necessary to solve a complex HJII, which is difficult to do analytically or numerically. Even if the HJII can be solved, it is impossible to implement this control method in the real world as this would require an infinite number of control devices to regulate the PDEs system, placed at all grid points of the global space domain simultaneously. A more feasible method is to regulate the PDEs system via a limited set of controllers placed at a selection of locations subject to environmental noise and system fluctuations. We combine fuzzy interpolation and finite difference schemes to approximate a nonlinear stochastic PDEs system. We then design a robust fuzzy tracking control based on this fuzzy spatial state space model, subject to unpredictable intrinsic and external disturbances and approximation errors.
With this control design procedure, the HJII can be transformed into an efficiently solvable LMIs problem. Based on this robust fuzzy tracking control design, the heterogeneous spatiotemporal distribution of natural resources, caused by factors such as stochastic disturbances, slope gradients, nutrient concentrations and species dispersal, can be adjusted to manage agricultural production, ecological conservation or ecosystem protection in the real world. Indeed, if the appropriate set of control devices exists, this method could be applied to almost any PDEs system.

This study demonstrates that ecological patterns in plant biomass distribution induced by environmental noise (spatiotemporal variation in nutrients, soil moisture and surface water) or spatial dynamics (diffusion, overland flow and root uptake) can be regulated to achieve a desired pattern using a limited set of control devices over the area being managed. If the effects of system spatial dynamics are uniform over each unit grid space, then control efforts via the limited set of control devices can be distributed over all the grid space to achieve a robust $\mathrm{H} \infty$ tracking control performance despite the effects of intrinsic parameter fluctuations and environmental disturbances. Our results show that tracking control performance increases with number of control devices but is also affected by spatial arrangement of devices. We can therefore use this method (subject to some restrictions) to determine the optimal number and arrangement of the control devices to achieve a prescribed tracking performance. This will be explored in future studies. This robust control strategy depends on the specified system 

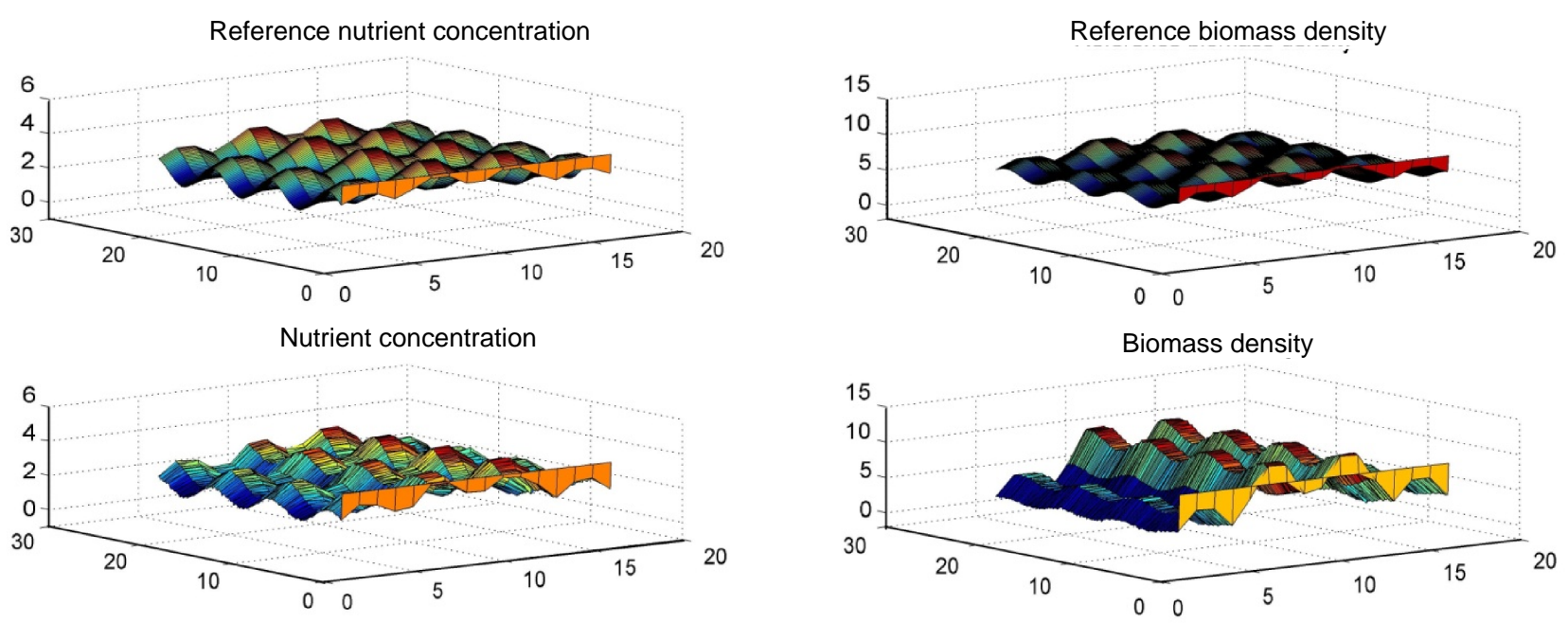

Tracking error of nutrient density

Tracking error of biomass density
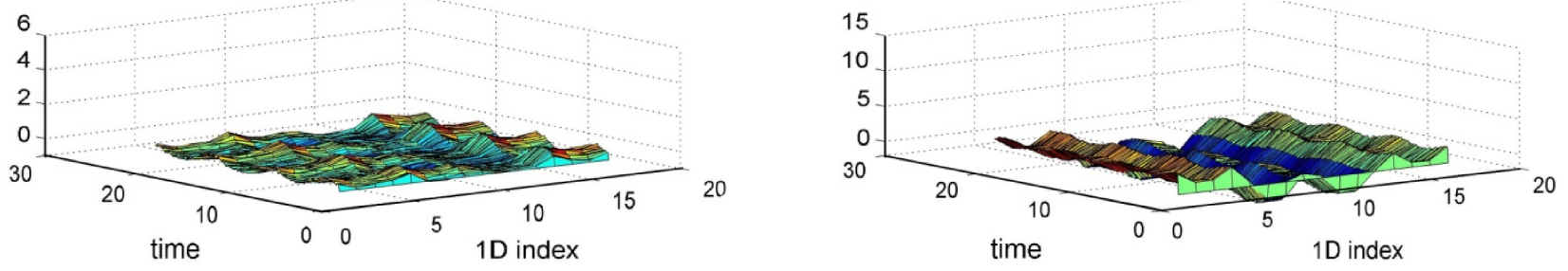

Figure 5. The spatiotemporal profiles of the optimal robust $\mathrm{H} \infty$ tracking control of the nonlinear stochastic nutrient-biomass system in (39) with control device distribution arrangement in Case 3 of Figure 6.

spatial dynamics, i.e. the diffusion and advection terms. These terms determine to what extent control interventions affect adjacent grid units, and therefore may limit the freedom of control design if diffusivity $D$ and velocity $V$ are weak. In future work, these spatial dynamics of an ecosystem may be included in the control design as follows: $u(x, t)=K_{1}(\tilde{\chi}(x, t))+$ $K_{2} \nabla(\tilde{\chi}(x, t))+K_{2} \nabla^{2}(\tilde{\chi}(x, t))$. In this way, the control strategy enhances not only the effects of the control intervention at control device locations but also the diffusion and advection of these effects into adjacent grid units. This may improve the efficiency of control interventions across the whole system.

Acknowledgements. This work was supported by the National Science Council of Taiwan under grant No. NSC 102-2745-E-007001-ASP

\section{Appendix}

Before the proof of Theorem 1, the following lemma is necessary.

Lemma A: For all vectors $X$ and $Y$ with appropriate dimensions, the following inequality always holds $X^{T} Y+Y^{T} X \leq$ $\xi X^{T} X+(1 / \xi) Y^{T} Y$ for any positive scalar value $\xi>0$.

\section{A. Proof of Theorem 1}

(i) Based on the augmented system in (11), let us denote a Lyapunov function $V(\bar{\chi}(x, t))>0$. From (12) or (13), we get:

$$
\begin{aligned}
& E \int_{0}^{t_{f}} \int_{\Omega}\left(\bar{\chi}(x, t)^{T} Q \bar{\chi}(x, t)\right) d x d t=E \int_{\Omega} V(\bar{\chi}(x, 0)) d x- \\
& E \int_{\Omega} V\left(\bar{\chi}\left(x, t_{f}\right)\right) d x+E \int_{0}^{t_{f}} \int_{\Omega}\left(\bar{\chi}(x, t)^{T} Q \bar{\chi}(x, t)+\frac{d V(\bar{\chi}(x, t))}{d t}\right) d x d t \\
& \leq E \int_{\Omega} V(\bar{\chi}(x, 0)) d x+E \int_{0}^{t_{f}} \int_{\Omega}\left(\bar{\chi}(x, t)^{T} Q \bar{\chi}(x, t)+\left(\frac{\partial V(\bar{\chi}(x, t))}{\partial \bar{\chi}(x, t)}\right)^{T}\right. \\
& (F(\bar{\chi}(x, t))+E v(x, t))+\frac{1}{2} F_{\omega}(\bar{\chi}(x, t))^{T}\left(\frac{\partial^{2} V(\bar{\chi}(x, t))}{\partial \bar{\chi}^{2}(x, t)}\right) \\
& \left.F_{\omega}(\bar{\chi}(x, t))\right) d x d t
\end{aligned}
$$

(by the fact that $E \int_{0} V\left(\bar{\chi}\left(x, t_{f}\right)\right) d x \geq 0, E d w(t)=0$ and Ito formula (Øksendal, 2003))

$$
\begin{aligned}
& \leq E \int_{\Omega} V(\bar{\chi}(x, 0)) d x+E \int_{0}^{t_{f}} \int_{\Omega}\left(\bar{\chi}(x, t)^{T} Q \bar{\chi}(x, t)+\left(\frac{\partial V(\bar{\chi}(x, t))}{\partial \bar{\chi}(x, t)}\right)^{T}\right. \\
& F(\bar{\chi}(x, t))+\frac{1}{4 \rho^{2}}\left(\frac{\partial V(\bar{\chi}(x, t))}{\partial \bar{\chi}(x, t)}\right)^{T} E E^{T}\left(\frac{\partial V(\bar{\chi}(x, t))}{\partial \bar{\chi}(x, t)}\right)+ \\
& \left.\rho^{2} v(x, t)^{T} v(x, t)+\frac{1}{2} F_{\omega}(\bar{\chi}(x, t))^{T}\left(\frac{\partial^{2} V(\bar{\chi}(x, t))}{\partial \bar{\chi}^{2}(x, t)}\right) F_{\omega}(\bar{\chi}(x, t))\right) d x d t
\end{aligned}
$$

(by Lemma A with $\xi=\rho^{2}$ ) 


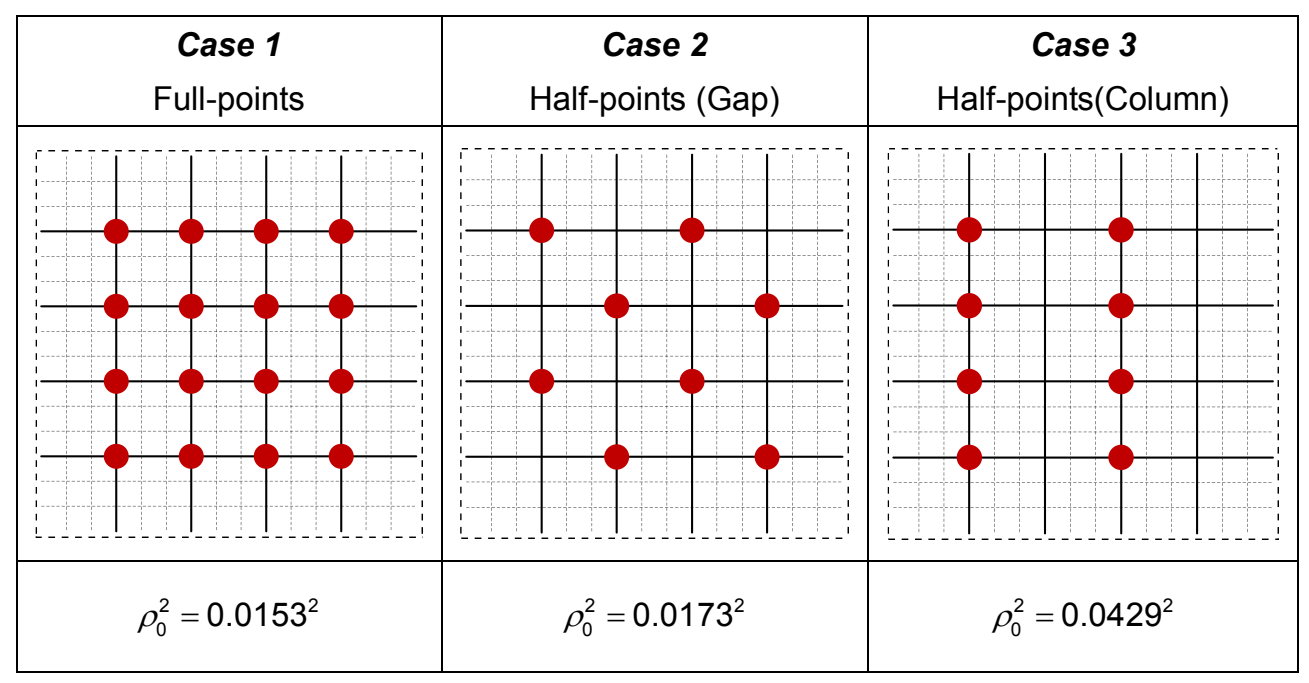

Figure 6. The optimal robust $\mathrm{H} \infty$ tracking control performance of three different distribution control strategies in 2 -D domain $(6 \times 6)$.

Suppose:

$$
\begin{aligned}
& \int_{\Omega}\left(\bar{\chi}(x, t)^{T} Q \bar{\chi}(x, t)+\left(\frac{\partial V(\bar{\chi}(x, t))}{\partial \bar{\chi}(x, t)}\right)^{T} F(\bar{\chi}(x, t))+\frac{1}{4 \rho^{2}}\right. \\
& \left(\frac{\partial V(\bar{\chi}(x, t))}{\partial \bar{\chi}(x, t)}\right)^{T} E E^{T}\left(\frac{\partial V(\bar{\chi}(x, t))}{\partial \bar{\chi}(x, t)}\right)+\frac{1}{2} F_{\omega}(\bar{\chi}(x, t))^{T} \\
& \left.\left(\frac{\partial^{2} V(\bar{\chi}(x, t))}{\partial \bar{\chi}^{2}(x, t)}\right) F_{\omega}(\bar{\chi}(x, t))\right) d x<0
\end{aligned}
$$

holds, then we have the following inequality:

$$
\begin{aligned}
& E \int_{0}^{t_{f}} \int_{\Omega}\left(\bar{\chi}(x, t)^{T} Q \bar{\chi}(x, t)\right) d x d t \leq E \int_{\Omega} V(\bar{\chi}(x, 0)) d x+ \\
& \rho^{2} E \int_{0}^{t_{f}} \int_{\Omega}\left(v(x, t)^{T} v(x, t)\right) d x d t
\end{aligned}
$$

Therefore, if the inequality in (15) holds, then the robust $\mathrm{H} \infty$ tracking control performance in (14) could be guaranteed. If $\bar{\chi}(x, 0)$, then $V(\bar{\chi}(x, 0))=0$ and the $\mathrm{H} \infty$ reference tracking performance in (13) holds.

(ii) If $v(x, t) \equiv 0$, then $E \int_{0}^{t_{f}} \int_{\Omega}\left(\bar{\chi}(x, t)^{T} Q \bar{\chi}(x, t)\right) d x d t \leq$ $E \int_{\Omega} V(\bar{\chi}(x, 0)) d x$. Since the $E \int_{\Omega} V(\bar{\chi}(x, 0)) d x$ is a finite value, as $t_{f} \rightarrow \infty, \tilde{\chi}(x, t)=\chi(x, t)-\chi_{r}(x, t)$ should asymptotically approach 0 in probability.

\section{B. Proof of Theorem 2}

(i) Based on the augmented system in (29), let us denote a Lyapunov function $V(\bar{\chi}(t))=\bar{\chi}(t)^{T} \bar{P} \bar{\chi}(t)>0$ as shown in (32). Thus, from (30), we get:

$E \int_{0}^{t_{f}} \bar{\chi}(t)^{T} \bar{Q} \bar{\chi}(t) d t=E V(\chi(0))-E V\left(\chi\left(t_{f}\right)\right)+E \int_{0}^{t_{f}}\left(\bar{\chi}(t)^{T} \bar{Q} \bar{\chi}(t)\right.$

$$
\begin{aligned}
& \left.+\frac{d \bar{\chi}(t)^{T} \bar{P} \bar{\chi}(t)}{d t}\right) d t \leq E V(\chi(0))+E \int_{0}^{t_{f}}\left\{\bar{\chi}(t)^{T} \bar{Q} \bar{\chi}(t) d t+\right. \\
& d \bar{\chi}(t)^{T} \bar{P} \bar{\chi}(t)+\bar{\chi}(t)^{T} \bar{P} d \bar{\chi}(t)+\sum_{i=1}^{L} \sum_{j=1}^{L} \overline{\bar{\mu}}_{i}(z(t)) \overline{\bar{\mu}}_{j}(z(t)) \\
& \left.\frac{1}{2}\left(\bar{\chi}(t)^{T} \bar{A}_{\bar{\omega} i}^{T} \frac{\partial^{2} \bar{\chi}(t)^{T} \bar{P} \bar{\chi}(t)}{d \bar{\chi}(t)^{2}} \bar{A}_{\bar{\omega} j} \bar{\chi}(t)\right) d t\right\}
\end{aligned}
$$

(by the fact that $E V\left(\chi\left(t_{f}\right)\right) \geq 0$ and Ito formula (Øksendal, 2003))

$$
\begin{aligned}
& =E V(\chi(0))+E \int_{0}^{t_{f}}\left\{\bar{\chi}(t)^{T} \bar{Q} \bar{\chi}(t)+\left(\sum_{i=1}^{L} \sum_{j=1}^{L} \overline{\bar{\mu}}_{i}(z(t)) \overline{\bar{\mu}}_{j}(z(t))\right.\right. \\
& \left.\left(\bar{A}_{i j} \bar{\chi}(t)+\bar{E} \bar{v}(t)+\bar{\varepsilon}(t)\right)\right)^{T} \bar{P} \bar{\chi}(t)+\bar{\chi}(t)^{T} \bar{P}\left(\sum_{i=1}^{L} \sum_{j=1}^{L} \overline{\bar{\mu}}_{i}(z(t))\right. \\
& \left.\overline{\bar{\mu}}_{j}(z(t))\left(\bar{A}_{i j} \bar{\chi}(t)+\bar{E} \bar{v}(t)+\bar{\varepsilon}(t)\right)\right)+\sum_{i=1}^{L} \sum_{j=1}^{L} \overline{\bar{\mu}}_{i}(z(t)) \overline{\bar{\mu}}_{j}(z(t)) \\
& \left.\frac{1}{2}\left(\left(\bar{\chi}(t)^{T} \bar{A}_{\bar{\omega} i}^{T}+\bar{\varepsilon}_{\bar{\omega}}(t)^{T}\right) \frac{\partial^{2} \bar{\chi}(t)^{T} \bar{P} \bar{\chi}(t)}{d \bar{\chi}(t)^{2}}\left(\bar{A}_{\bar{\omega} j} \bar{\chi}(t)+\bar{\varepsilon}_{\bar{\omega}}(t)\right)\right)\right\} d t
\end{aligned}
$$

(by the fact that $E d w(t)=0$ )

$$
\begin{aligned}
& =E V(\chi(0))+E \int_{0}^{t_{f}}\left\{\sum _ { i = 1 } ^ { L } \sum _ { j = 1 } ^ { L } \overline { \overline { \mu } } _ { i } ( z ( t ) ) \overline { \overline { \mu } } _ { j } ( z ( t ) ) \left(\overline { \chi } ( t ) ^ { T } \left(\bar{A}_{i j}^{T} \bar{P}+\right.\right.\right. \\
& \left.\bar{P} \bar{A}_{i j}+\bar{Q}+\bar{P} \bar{P}+\bar{\Sigma}^{T} \bar{\Sigma}+2\left(\bar{A}_{\bar{\omega} i}+\bar{\Sigma}_{\bar{\omega}}\right)^{T} \bar{P}\left(\bar{A}_{\bar{\omega} j}+\bar{\Sigma}_{\bar{\omega}}\right)\right) \bar{\chi}(t) \\
& \left.\left.+\bar{v}(t)^{T} \bar{E}^{T} \bar{P} \bar{\chi}(t)+\bar{\chi}(t)^{T} \bar{P} \bar{E} \bar{v}(t)\right)\right\} d t \\
& \leq E V(\chi(0))+E \int_{0}^{t_{f}}\left\{\sum _ { i = 1 } ^ { L } \sum _ { j = 1 } ^ { L } \overline { \overline { \mu } } _ { i } ( z ( t ) ) \overline { \overline { \mu } } _ { j } ( z ( t ) ) \left(\overline { \chi } ( t ) ^ { T } \left(\bar{A}_{i j}^{T} \bar{P}+\right.\right.\right.
\end{aligned}
$$


$\bar{P} \bar{A}_{i j}+\bar{Q}+\bar{P} \bar{P}+\bar{\Sigma}^{T} \bar{\Sigma}+2\left(\bar{A}_{\bar{\omega} i}+\bar{\Sigma}_{\bar{\omega}}\right)^{T} \bar{P}\left(\bar{A}_{\bar{\omega} j}+\bar{\Sigma}_{\bar{\omega}}\right)+$

$\left.\left.\frac{1}{\rho^{2}} \bar{P} \bar{E} \bar{E}^{T} \bar{P}\right) \bar{\chi}(t)+\rho^{2} \bar{v}(t)^{T} \bar{v}(t)\right\} d t$

Because by Lemma A and Assumption 1, we have:

$\bar{\varepsilon}(t)^{T} \bar{P} \bar{\chi}(t)+\bar{\chi}(t)^{T} \bar{P} \bar{\varepsilon}(t) \leq \bar{\varepsilon}(t)^{T} \bar{\varepsilon}(t)+\bar{\chi}(t)^{T} \bar{P} \bar{P} \bar{\chi}(t) \leq$

$\bar{\chi}(t)^{T} \bar{\Sigma}^{T} \bar{\Sigma} \bar{\chi}(t)+\bar{\chi}(t)^{T} \overline{P P} \bar{\chi}(t)$

$\left(\bar{\chi}(t)^{T} \bar{A}_{\bar{\omega} i}^{T}+\bar{\varepsilon}_{\bar{\omega}}(t)^{T}\right) \bar{P}\left(\bar{A}_{\bar{\omega} j} \bar{\chi}(t)+\bar{\varepsilon}_{\bar{\omega}}(t)\right) \leq 2\left(\bar{\chi}(t)^{T} \bar{A}_{\bar{\omega} i}^{T} \bar{P}_{\bar{\omega} j} \bar{\chi}(t)+\right.$ $\left.\bar{\chi}(t)^{T} \bar{\Sigma}_{\bar{\omega}}^{T} \bar{P}_{\bar{\omega}} \bar{\chi}(t)\right)$

where $\bar{\Sigma}=\left[\begin{array}{ll}0 & \delta I\end{array}\right], \bar{\Sigma}_{\bar{\omega}}=\operatorname{diag}\left(0, \delta_{\omega} I\right)$.

Suppose:

$\bar{A}_{i j}^{T} \bar{P}+\bar{P}_{i j}+\bar{Q}+\bar{P} \bar{P}+\bar{\Sigma}^{T} \bar{\Sigma}+2\left(\bar{A}_{\bar{\omega} i}+\bar{\Sigma}_{\bar{\omega}}\right)^{T} \bar{P}\left(\bar{A}_{\bar{\omega} j}+\bar{\Sigma}_{\bar{\omega}}\right)+$

$\frac{1}{\rho^{2}} \bar{P} \bar{E} \bar{E}^{T} \bar{P}<0$

or the equivalent matrix inequalities

$$
\left[\begin{array}{ccc}
\bar{A}_{i j}^{T} \bar{P}+\bar{P}_{i j}+\bar{Q}+\bar{\Sigma}^{T} \bar{\Sigma}+2\left(\bar{A}_{\bar{\omega} i}+\bar{\Sigma}_{\bar{\omega}}\right)^{T} \bar{P}\left(\bar{A}_{\bar{\omega} j}+\bar{\Sigma}_{\bar{\omega}}\right) & \bar{P} \bar{E} & \bar{\Sigma}^{T} \\
\bar{E}^{T} \bar{P} & -\rho^{2} I & 0 \\
\bar{\Sigma} & 0 & -I
\end{array}\right]
$$

$\leqslant 0$

hold, we have the following inequality:

$$
E \int_{0}^{t_{f}} \bar{\chi}(t)^{T} \bar{Q} \bar{\chi}(t) d t \leq E V(\chi(0))+\rho^{2} E \int_{0}^{t_{f}} \bar{v}(t)^{T} \bar{v}(t) d t
$$

Therefore, if the inequality in (35) holds, then the $\mathrm{H} \infty$ reference tracking performance in (32) could be guaranteed. If $\chi(0) \equiv 0$, then the $\mathrm{H} \infty$ reference tracking performance in (31) holds.

(ii) The proof is the same as that in Theorem 1 .

\section{Proof of Theorem 3}

(i) The fact that the inequality in (36) implies the inequality in (34) is proven in the following.

By Schur complement (Boyd et al., 1994), the inequality in (34) is equivalent to:

$$
\begin{aligned}
& \bar{A}_{i j}^{T} \bar{P}+\bar{P}_{i j}+\bar{Q}+\bar{P} \bar{P}+\bar{\Sigma}^{T} \bar{\Sigma}+2\left(\bar{A}_{\bar{\omega} i}+\bar{\Sigma}_{\bar{\omega}}\right)^{T} \bar{P}\left(\bar{A}_{\bar{\omega} j}+\bar{\Sigma}_{\bar{\omega}}\right)+\frac{1}{\rho^{2}} \bar{P} \bar{E} \bar{E}^{T} \bar{P} \\
& <0
\end{aligned}
$$

By pro- and post-multiplying $\bar{X}$ on the above inequality, we can obtain the following result:

$$
\begin{aligned}
& \bar{X}\left(\bar{A}_{i j}^{T} \bar{P}+\bar{P} \bar{A}_{i j}+\bar{Q}+\bar{P} \bar{P}+\bar{\Sigma}^{T} \bar{\Sigma}+2\left(\bar{A}_{\bar{\omega} i}+\bar{\Sigma}_{\bar{\omega}}\right)^{T} \bar{P}\left(\bar{A}_{\bar{\omega} j}+\bar{\Sigma}_{\bar{\omega}}\right)+\right. \\
& \left.\frac{1}{\rho^{2}} \bar{P} \bar{E} \bar{E}^{T} \bar{P}\right) \bar{X}=\bar{X}_{i j}^{T}+\bar{A}_{i j} \bar{X}+\bar{X} \bar{Q} \bar{X}+\bar{I}+2 \bar{X}\left(\bar{A}_{\bar{\omega} i}+\bar{\Sigma}_{\bar{\omega}}\right)^{T} \bar{X}^{-1} \\
& \left(\bar{A}_{\bar{\omega} j}+\bar{\Sigma}_{\bar{\omega}}\right) \bar{X}+\frac{1}{\rho^{2}} \bar{E} \bar{E}^{T}<0
\end{aligned}
$$

By Schur's complement (Boyd et al., 1994), we obtain following LMIs:

$$
\left[\begin{array}{ccccc}
\bar{X}_{i j}^{T}+\bar{A}_{i j} \bar{X}+\bar{I} & \bar{X} \bar{Q}_{1 / 2}^{T} & \bar{X}\left(\bar{A}_{\bar{\omega} j}+\bar{\Sigma}_{\bar{\omega}}\right)^{T} & \bar{E} & \bar{X} \bar{\Sigma}^{T} \\
\bar{Q}_{1 / 2} \bar{X} & -I & 0 & 0 & 0 \\
\left(\bar{A}_{\bar{\omega} i}+\bar{\Sigma}_{\bar{\omega}}\right) \bar{X} & 0 & -\frac{1}{2} \bar{X} & 0 & 0 \\
\bar{E}^{T} & 0 & 0 & -\rho^{2} I & 0 \\
\bar{\Sigma} \bar{X} & 0 & 0 & 0 & -I
\end{array}\right]<0
$$

Using the results, i.e. the inequality in (36) implies the inequality in (34), we can get that Theorem 3 implies the Theorem 2 , i.e. if the inequality in (36) holds then the $\mathrm{H} \infty$ reference tracking control performance in (31) can be guaranteed by the fuzzy tracking controller in (28).

(ii) The proof is the same as the proof in Theorem 1

\section{References}

Ahuja, L.R., Reddy, V.R., Saseendran, S.A., and Qiang, Y. (2008). Response of Crops to Limited Water: Understanding and Modeling Water Stress Effects on Plant Growth Processes, American Society of Agronomy Inc, Madison.

Amir, J., Krikun, J., Orion, D., Putter, J., and Klitman, S. (1991). Wheat production in an arid environment. 1. Water-use efficiency, as affected by management practices. Field Crops Res., 27, 351-364. http://dx.doi.org/10.1016/0378-4290(91)90041-S

Batchelor, C., Lovell, C., and Murata, M. (1996). Simple microirrigation techniques for improving irrigation efficiency on vegetable gardens. Agric. Water Manage., 32, 37-48. http://dx.doi.org/10.101 6/S0378-3774(96)01257-7

Bergen, S.D., Bolton, S.M., and Fridley, J.L. (2001). Design principles for ecological engineering. Ecol. Eng., 18, 201-210. http://dx. doi.org/10.1016/S0925-8574(01)00078-7

Bouman, B., Peng, S., Castaneda, A., and Visperas, R. (2005). Yield and water use of irrigated tropical aerobic rice systems. Agric. Water Manage., 74, 87-105. http://dx.doi.org/10.1016/j.agwat.2004. 11.007

Boutraa, T. (2010). Improvement of water use efficiency in Irrigated agriculture: A Review. J. Agron., 9, 1-8. http://dx.doi.org/10.3923/ ja.2010.1.8

Boyd, S., El Ghaoui, L., Feron, E., and Balakrishnan, V. (1994). Linear Matrix Inequalities in System and Control Theory, SIAM: Philadelphia, PA. USA. http://dx.doi.org/10.1137/1.9781611970777

Burke, S., Mulligan, M., and Thornes, J.B. (1999). Optimal irrigation efficiency for maximum plant productivity and minimum water loss. Agric. Water Manage., 40, 377-391. http://dx.doi.org/10.1016/ 
S0378-3774(99)00011-6

Cale, P., Allen-Diaz, B.H., Hobbs, R.J., and Suding, K.N. (2009). New Models for Ecosystem Dynamics and Restoration, Island Press, New York.

Chang, Y.T., and Chen, B.S. (2010). A fuzzy approach for robust reference-tracking-control design of nonlinear distributed parameter time-delayed systems and its application. IEEE Trans. Fuzzy Syst., 18, 1041-1057. http://dx.doi.org/10.1109/TFUZZ.2010.2058809

Chen, B.S., and Chang, Y.T. (2009). Fuzzy state-space modeling and robust observer-based control design for nonlinear partial differential systems. IEEE Trans. Fuzzy Syst., 17, 1025-1043. http://dx.doi. org/10.1109/TFUZZ.2009.2020506

Chen, B.S., Chen, W.H., and Wu, H.L. (2009). Robust H2/Ho global linearization filter design for nonlinear stochastic systems. IEEE Trans. Circuits Syst., 56, 1441-1454. http://dx.doi.org/10.110 9/TCSI.2008.2007059

Chen, B.S., Chen, W.H., and Zhang, W. (2012). Robust filter for nonlinear stochastic partial differential systems in sensor signal processing: Fuzzy approach. IEEE Trans. Fuzzy Syst., 20, 957-970. http://dx.doi.org/10.1109/TFUZZ.2012.2190516

Chen, B.S., Lee, C.H., and Chang, Y.C. (1996). Hळ tracking design of uncertain nonlinear SISO systems: Adaptive fuzzy approach. IEEE Trans. Fuzzy Syst., 4, 32-43. http://dx.doi.org/10.1109/91.48 1843

Chen, B.S., Tseng, C.S., and Uang, H.J. (1999). Robustness design of nonlinear dynamic systems via fuzzy linear control. IEEE Trans. Fuzzy Syst., 7, 571. http://dx.doi.org/10.1109/91.797980

Chen, B.S., Tseng, C.S., and Uang, H.J. (2000). Mixed H2/H infinity fuzzy output feedback control design for nonlinear dynamic systems: An LMI approach. IEEE Trans. Fuzzy Syst., 8(3), 249-265. http://dx.doi.org/10.1109/91.85591

Chen, Y., Cheng, S., Liu, L., Guo, X., Wang, Z., Qin, C., Hao, R., Lu, J., and Gao, J. (2013). Assessing the effects of land use changes on non-point source pollution reduction for the Three Gorges Watershed using the SWAT model. J. Environ. Inf., 22, 13-26. http://dx. doi.org/10.3808/jei.201300242

Evans, G., Blackledge, J.M., and Yardley, P. (2000). Numerical Methods for Partial Differential Equations, Springer Verlag, London. http://dx.doi.org/10.1007/978-1-4471-0377-6

Evans, R.O., Sneed, R.E., Cassel, D.K., and Service, N.C.C.E. (1991). Irrigation Scheduling to Improve Water-and Energy-use Efficiencies, NC Cooperative Extension Service.

FAO (2003). Agriculture, Food and Water.

Fereres, E., and Soriano, M.A. (2007). Deficit irrigation for reducing agricultural water use. J. Exp. Bot., 58, 147-159. http://dx.doi.org/ $10.1093 / \mathrm{jxb} / \mathrm{erl} 165$

Gurney, W., and Nisbet, R.M. (1998). Ecological Dynamics, Oxford University Press, Oxford.

Hanjra, M.A., and Qureshi, M.E. (2010). Global water crisis and future food security in an era of climate change. Food Policy, 35, 365-377. http://dx.doi.org/10.1016/j.foodpol.2010.05.006

Huang, D., and Nguang, S.K. (2006). Robust static output feedback control of fuzzy systems: An ILMI approach. IEEE Trans. Syst. Man Cybernetics, Pt B: Cybernetics, 36, 217. http://dx.doi.org/ 10.1109/TSMCB.2005.856145

Huang, Y., and Diekmann, O. (2003). Interspecific influence on mobility and Turing instability. Bull. Math. Biol., 65, 143-156. http://dx. doi.org/10.1006/bulm.2002.0328

Klausmeier, C.A. (1999). Regular and irregular patterns in semiarid vegetation. Science, 284, 1826. http://dx.doi.org/10.1126/science. 284.5421.1826

Kocvara, M., and Stingl, M. (2003). PENNON: A code for convex nonlinear and semidefinite programming. Optimiz. Meth. Softw., 18, 317-334. http://dx.doi.org/10.1080/1055678031000098773
Matthews, G.A. (1999). Application of Pesticides to Crops, Imperial College Pr. http://dx.doi.org/10.1142/p144

Monsef, H., and Abahussain, A. (2013). The impact of camping activities on soil degradation in Kuwait. J. Environ. Inf., 22, 102-111. http://dx.doi.org/10.3808/jei.201300249

Nguang, S.K., and Shi, P. (2003). Ho fuzzy output feedback control design for nonlinear systems: An LMI approach. IEEE Trans. Fuzzy Syst., 11, 331-340. http://dx.doi.org/10.1109/TFUZZ.2003. 812691

Øksendal, B. (2003). Stochastic Differential Equations, Springer Verlag, Berlin. http://dx.doi.org/10.1007/978-3-642-14394-6

Pearce, D., and Koundouri, P. (2003). Fertilizer and pesticide taxes for controlling non-point agricultural pollution. Agric. Rural Develop., 1.

Recknagel, F. (2013). Current scope, case studies and future directions of ecological informatics. J. Environ. Inf., 21, 3-11. http://dx.doi. org/10.3808/jei.201300227

Rosegrant, M.W., Cai, X., and Cline, S.A. (2002). World Water and Food to 2025: Dealing with Scarcity, International Food Policy Research Institute.

Satnoianu, R.A., Menzinger, M., and Maini, P.K. (2000). Turing instabilities in general systems. J. Math. Biol., 41, 493-512. http://dx. doi.org/10.1007/s002850000056

Strikwerda, J.C. (2004). Finite Difference Schemes and Partial Differential Equations, Society for Industrial and Applied Mathematics (SIAM), Philadelphia. http://dx.doi.org/10.1137/1.9780898717938

Takagi, T., and Sugeno, M. (1985). Fuzzy identification of system and its applications to modelling and control. IEEE Trans. Syst. Man Cybernetics, 15, 116-132. http://dx.doi.org/10.1109/TSMC. 1985.6313399

Tanaka, K., Ikeda, T., and Wang, H.O. (1996). Robust stabilization of a class of uncertain nonlinear systems via fuzzy control: Quadratic stabilizability, $\mathrm{H} \infty$ control theory, and linear matrix inequalities. IEEE Trans. Fuzzy Syst., 4, 1-13. http://dx.doi.org/10.1109/91.48 1840

Tanaka, K., Ikeda, T., and Wang, H.O. (1998). Fuzzy regulators and fuzzy observers: Relaxed stability conditions and LMI-based designs. IEEE Trans. Fuzzy Syst., 6, 250-265. http://dx.doi.org/10.110 9/91.669023

Tanaka, K., and Sugeno, M. (1992). Stability analysis and design of fuzzy control systems. Fuzzy Sets Syst., 45, 135-156. http://dx.doi. org/10.1016/0165-0114(92)90113-I

Tanaka, K., and Wang, H.O. (2004). Fuzzy Control Systems Design and Analysis: A Linear Matrix Inequality Approach, Wiley, New York.

Thébault, E., and Fontaine, C. (2010). Stability of ecological communities and the architecture of mutualistic and trophic networks. Science, 329, 853-856. http://dx.doi.org/10.1126/science.1188321

Tilman, D., Cassman, K.G., Matson, P.A., Naylor, R., and Polasky, S. (2002). Agricultural sustainability and intensive production practices. Nature, 418, 671-677. http://dx.doi.org/10.1038/nature01014

Tseng, C.S., Chen, B.S., and Uang, H.J. (2001). Fuzzy tracking control design for nonlinear dynamic systems via TS fuzzy model. IEEE Trans. Fuzzy Syst., 9, 381-392. http://dx.doi.org/10.1109/91. 928735

USCB (2011). International Data Base World Population: 1950-2050.

Wallace, J. (2000). Increasing agricultural water use efficiency to meet future food production. Agric. Ecosyst. Environ., 82, 105-119. http://dx.doi.org/10.1016/S0167-8809(00)00220-6

WBCSD and IUCN (2009). Agricultural Ecosystems: Facts and Tren$d s$, WBCSD and IUCN, Gland.

Wu, H.N., and Li, H.X. (2008). Ho fuzzy observer-based control for a class of nonlinear distributed parameter systems with control constraints. IEEE Trans. Fuzzy Syst., 16, 502-516. http://dx.doi.org/10. 
1109/TFUZZ.2007.896351

$\mathrm{Xu}, \mathrm{T}$, and Qin, X. (2013). Solving water quality management problem through combined genetic algorithm and fuzzy simulation. $J$. Environ. Inf., 22, 39-48. http://dx.doi.org/10.3808/jei.201300244

Yang, Y., and Chen, H. (2013). Assessing impacts of flow regulation on trophic interactions in a wetland ecosystem. J. Environ. Inf., 21, 63-71. http://dx.doi.org/10.3808/jei.201300233
Yoo, H. (2000). Semi-discretization of stochastic partial differential equations on R1 by a finite-difference method. Math. Computation, 69, 653-666. http://dx.doi.org/10.1090/S0025-5718-99-01150-3

Yuan, K., Li, H.X., and Cao, J. (2008). Robust stabilization of the distributed parameter system with time delay via fuzzy control. IEEE Trans. Fuzzy Syst., 16, 567-584. http://dx.doi.org/10.1109/TFUZZ. 2007.896233 